\title{
Lattice investigation of exotic tetraquark channels
}

\author{
R. J. Hudspith $\odot,{ }^{1, *}$ B. Colquhoun $\odot,{ }^{2}$ A. Francis, ${ }^{3}$ R. Lewis, ${ }^{2}$ and K. Maltman $\odot^{4}$ \\ ${ }^{1}$ PRISMA ${ }^{+}$Cluster of Excellence and Institut für Kernphysik, Johannes Gutenberg-Universität Mainz, \\ D-55099 Mainz, Germany \\ ${ }^{2}$ Department of Physics and Astronomy, York University, Toronto, Ontario, M3J 1P3, Canada \\ ${ }^{3}$ Theoretical Physics Department, CERN, CH-1211 Geneva 23, Switzerland \\ ${ }^{4}$ Department of Mathematics and Statistics, York University, Toronto, Ontario M3J 1P3, Canada \\ and CSSM, University of Adelaide, Adelaide, South Australia 5005, Australia
}

(Received 8 July 2020; accepted 27 October 2020; published 8 December 2020)

\begin{abstract}
We perform an $n_{f}=2+1$ lattice study of a number of channels where past claims exist in the literature for the existence of strong-interaction-stable light-heavy tetraquarks. We find no evidence for any such deeply bound states, beyond the $J^{P}=1^{+}, I=0 u d \bar{b} \bar{b}$ and $I=1 / 2 \ell s \bar{b} \bar{b}$ states already identified in earlier lattice studies. We also describe a number of systematic improvements to our previous lattice studies, including working with larger $m_{\pi} L$ to better suppress possible finite volume effects, employing extended sinks to better control excited-state contamination, and expanding the number of operators used in the GEVP analyses. Our results also allow us to rule out several phenomenological models which predict significant tetraquark binding in channels where no such binding is found.
\end{abstract}

DOI: 10.1103/PhysRevD.102.114506

\section{INTRODUCTION}

Results from multiple recent lattice studies [1-5] now rather firmly establish the existence of an exotic, doubly bottom, $I=0, J^{P}=1^{+} u d \bar{b} \bar{b}$ tetraquark state, bound with respect to $B B^{*}$, and hence strong-interaction stable. Though results for the binding energy vary, all correspond to masses below $B B$ threshold, making the state stable with respect to, not only strong-interaction, but also electromagnetic, decays. The results of Refs. [3,4] also predict a strong-interaction-stable flavor $\overline{3}_{F}, I=1 / 2, J^{P}=1^{+}$ strange partner. References [6,7], using heavy quark symmetry arguments, supplemented by either phenomenological input [6] or phenomenological plus lattice input [7] for leading finite heavy mass corrections, concur on the strong-interaction stability of both the $I=0$ and $I=1 / 2$ doubly bottom states [8]. The binding in these channels appears to be driven by a combination of the attractive short-distance color Coulomb interaction between two $\bar{b}$ antiquarks in a color $3_{c}$ and the spin-dependent interaction of light quarks in the $\overline{3}_{F}$, spin 0 , color $\overline{3}_{c}$ "good light diquark" configuration, whose attractive character is known phenomenologically from observed heavy baryon splittings

\footnotetext{
*renwick.james.hudspith@gmail.com
}

Published by the American Physical Society under the terms of the Creative Commons Attribution 4.0 International license. Further distribution of this work must maintain attribution to the author(s) and the published article's title, journal citation, and DOI. Funded by SCOAP.
$[3,9]$. Neither of these interactions is accessible in a state consisting of two well-separated heavy mesons.

While the existence of this $\overline{3}_{F}$ of $J^{P}=1^{+}$stronginteraction-stable tetraquark states seems well established theoretically, detecting these states, and confirming this prediction, remains experimentally challenging. Production rates are likely to be very low, given that two $b \bar{b}$ pairs must first be produced. While the doubly charmed $\Xi_{c c}$ baryon has now been observed by LHCb $[10,11]$, and double- $\Upsilon$ production has been reported by CMS [12], to date, not even bottom-charm, let alone doubly bottom baryon states have been detected. The recently proposed inclusive search strategy, in which a $B_{c}$ meson originating from a displaced vertex would signal the presence of doubly bottom hadron production at the LHC [13], though clearly of interest, would still leave open the question of whether such a signal contained a doubly bottom tetraquark component or was due entirely to doubly bottom baryon production.

In this paper we use lattice QCD calculations to investigate whether strong-interaction-stable (hereafter "bound") tetraquarks exist in other channels likely to be more amenable to experimental detection. A number of such channels have been investigated in the literature using various approaches, including QCD-inspired models [14-42] and QCD sum rules [43-55], and we will study a range of channels where claims for the existence of such bound tetraquark states have been made. Our investigations will also serve to test those aspects of the models not constrained by fits to the ordinary meson and baryon spectrum as well as approximations employed in implementing the QCD sum rule framework in studies of tetraquark channels. 
In the case of model studies, the parameters of the models (most typically constituent quark models) are fixed from earlier fits to the ordinary meson and baryon spectrum. Interactions of a constituent quark pair in a color $\overline{3}_{c}$ or a constituent quark and antiquark in a color $1_{c}$ configuration are, thus, phenomenologically constrained. In tetraquark (and other multiquark) channels, however, additional color configurations, which have totally unconstrained constituent interactions, are also present. In those channels already identified by the lattice as supporting deeply bound, doubly bottom tetraquark states, where the physics of the binding is believed to be dominated by a combination of the color Coulomb attraction in the antidiquark color $3_{c}$ and the diquark attraction in the good-light-diquark $\overline{3}_{c}$ configuration, these dominant interactions are phenomenologically constrained, and one would, therefore, expect the models to successfully predict tetraquark binding in these channels. This is, indeed, typically the case. However, even in these channels, differences in the assumed form of the model interactions, which, by construction, produce no differences in the ordinary hadron spectrum (since the model parameters have been fit to ensure the spectrum is reproduced) can produce significant differences in an exotic channel. An example is provided by the comparison of results for predicted binding in the $I=0, J^{P}=1^{+} u d \bar{b} \bar{b}$ channel produced by a range of dynamical chiral [21,23,25, $26,29,30,36,41]$ and nonchiral [16,17,19,20,22,24,26,27, $29-32,37,40,42]$ quark models. The latter typically employ a purely one-gluon-exchange (OGE) form for the spin-spin interaction, while spin-spin interactions for the former are produced by a combination of effective Goldstone-boson exchange, acting between the light constituents only, and nominal OGE acting between all constituents. Even if the tetraquark state is entirely dominated by the $3_{c}$ antidiquark, $\overline{3}_{c}$ good-light-diquark configuration, this configuration includes both $1_{c}$ and $8_{c} \bar{b}$-light quark pairs. The fits of the models to the ordinary meson and baryon spectrum place no constraints on these residual $8_{c}$ heavy-light interactions. In addition, the color dependence of the OGE interaction and the absence of Goldstone-boson-exchange contributions to the spin-dependent heavy-light interactions in the chiral quark model framework mean one must expect significant differences in the residual heavy-light interactions and hence in the predictions for the tetraquark binding, in the two classes of models. This expectation is also borne out in the literature, where the dynamical nonchiral models of Refs. [16,17,19,20,22,24,27,29-31,37,40,42] produce binding energies between 54 and $160 \mathrm{MeV}$, in contrast to the results of the dynamical chiral models of Refs. [21,23,29, 30,36,41], which lie between 214 and $497 \mathrm{MeV}$.

Different systematic questions arise for predictions generated using QCD sum rules, where the underlying dispersive representations are rigorously valid but practical applications require approximations on both the spectral and OPE sides. All tetraquark studies we are aware of work with Borel-transformed sum rules and the SVZ spectral ansatz, which consists of a single low-lying "pole" (characterized by its mass, $M$, and coupling, $f$, to the relevant interpolating current) and a continuum [approximated using the operator product expansion (OPE)] for $s$ above a "continuum threshold" $s_{0}$ [56,57]. The OPE side is taken as input, and the sum rules used to fix $M, f$, and $s_{0}$. The OPE, however, involves not just known quantities, like quark masses, $\alpha_{s}$ and $\langle\bar{q} q\rangle$, but also unknown higher dimension condensates. Contributions up to dimensions $D=8$ or 10 are retained in recent tetraquark analyses. As stressed in the earliest of the sum rule studies of doubly heavy tetraquarks [43], estimating such higher $D$ contributions (typically in terms of products of known lower-dimension condensates) requires use of the factorization approximation. The accuracy of this approximation is not known in general, but for the $I=1$ vector and axial-vector current-current two-point functions, extractions of the two relevant $D=6$ four-quark condensates from finite-energy sum rule analyses of OPAL [58] and ALEPH [59] hadronic $\tau$ decay data found it to be off by as much as a factor of $\sim 6[60,61]$. On the spectral side of the sum rules, the single-narrow-resonance-plus-continuum SVZ ansatz may not be suitable for all channels. It would, for example, represent a poor choice for the $I=0, J^{P}=0^{-}$ channel, which has not one, but three narrow, low-lying $\eta$ resonances. Its use in exotic channels, where limited prior knowledge of the qualitative features of the spectral distribution is likely, thus has the potential to produce difficultto-assess systematic uncertainties. One way to investigate this issue is to consider sum rules for different interpolating operators with the same quantum numbers. The associated spectral functions will all receive contributions from all states with these quantum numbers, with only the contribution strengths operator dependent. Finding compatible pole masses and similar continuum thresholds from all the sum rules would increase confidence in the reliability of the SVZ ansatz. Finding incompatible pole masses would, in contrast, signal either that the approximations used on the OPE sides were unreliable or that the channel has more than one narrow state, raising questions about the suitability of the use of the SVZ ansatze.

The lattice approach is ideally suited to investigating channels with a deeply bound tetraquark ground state and to testing model and sum rule predictions for the existence of such states. Provided one employs interpolating operators with reasonable ground state overlap, the sizeable gap to the lowest meson-meson threshold increases the likelihood the ground state will dominate the corresponding two-point function at moderate Euclidean times, before the signal is lost in noise. Judicious source and sink choices (discussed in more detail in Sec. IV below) are also relevant to improving the ground state signal. Weakly bound states represent more of a challenge because finite volume (FV) effects have the potential to produce a FV analogue of what will become a meson-meson scattering state in the 
continuum that is shifted below the continuum mesonmeson threshold. FV effects are expected to be small for bound states, but not necessarily negligible for continuum scattering states. They are typically handled either by extrapolations using simulations at multiple volumes or by working with ensembles where the product $m_{\pi} L$, with $L$ the lattice length, is large enough to suppress the dominant "round-the-world" FV effects. A general rule of thumb is that this requires $m_{\pi} L>4$. The fact that the good-lightdiquark configuration is believed to play an important role in binding for the tetraquarks so far identified and that, phenomenologically, the associated contributions to binding are expected to grow with decreasing light quark mass, also puts a premium on working with $m_{\pi}$ as close to the physical point as possible, subject to maintaining a detectable signal and keeping FV effects under control.

For the $32^{3} \times 64$ ensembles used in our previous studies of the $J^{P}=1^{+}, \overline{3}_{F} u d \bar{b} \bar{b}$ and $\ell s \bar{b} \bar{b}(\ell=u, d)$ channels [3] and $J^{P}=1^{+}, I=0 u d \bar{c} \bar{b}$ channel [9] the $m_{\pi} L$ values were 6.1 for $m_{\pi} \simeq 415 \mathrm{MeV}$ and 4.4 for $m_{\pi} \simeq 299 \mathrm{MeV}$ but only 2.4 for $m_{\pi} \simeq 164 \mathrm{MeV}$. For the current study, which focuses on searching for channels with bound, nonmolecular (i.e., nonweakly bound) tetraquark ground states, we have thus generated a new ensemble with the same lattice spacing but a larger volume $\left(48^{3} \times 64\right)$ and a pion mass, $m_{\pi} \simeq 192 \mathrm{MeV}$, still close to physical, but with $m_{\pi} L=4.2>4$. With only this single $m_{\pi}$, we will be unable to identify channels in which a shallow bound state might exist at physical $m_{\pi}$, but not at the slightly higher $m_{\pi}$ of our simulation. Channels identified as having a moderate-to-deeply bound tetraquark ground state for $m_{\pi} \simeq 192 \mathrm{MeV}$, will, however, certainly also have such a ground state at slightly lower, physical $m_{\pi}$.

We now turn to the channels to be investigated in this paper. The likelihood of binding is increased by restricting our attention to those channels where no relative spatial excitations are required and where light quark pairs have access to the favorable $\overline{3}_{F}$, spin 0 , color $\overline{3}_{c}$, good-lightdiquark configuration. The antiquark pair must then have access to the $3_{c}$ configuration and, with no relative spatial excitation, have spin 1 if the two antiquarks are the same. Both spin 0 and 1 are possible if the antiquarks are different. These considerations lead us to investigate channels with either $I=0$ or $1 / 2, J^{P}=1^{+}$and two identical antiquarks, or $I=0$ or $1 / 2, J^{P}=0^{+}$or $1^{+}$and two nonidentical antiquarks.

In what follows, we will first briefly revisit our previous study [3] of the doubly bottom, $J^{P}=1^{+}, \overline{3}_{F}$ channels already well established to support bound tetraquark states. Our goal here is not to reestablish this fact, but rather to illustrate an important improvement to the methods employed in Ref. [3]. In that analysis (as well as in our analysis of the $I=0, J^{P}=1^{+} u d \bar{c} \bar{b}$ channel [9]) local sinks were combined with gauge-fixed wall sources. With this setup, the resulting ground state effective mass plateaus were short and reached at later Euclidean times. With wall-local effective masses typically plateauing from below, this leaves open the possibility that the true plateaus had not yet been reached and the resulting binding energies overestimated. Improving the ground state signal is thus desirable, and we have succeeded in accomplishing this goal using the extended "box-sink" construction, described in Sec. IV below. The results for the bound doubly bottom $\overline{3}_{F}$ ground states, at the $m_{\pi}$ of this study, are thus included primarily to illustrate this improvement, and to motivate the use of the box-sink construction in the other channels considered here which are identified and discussed in more detail in Sec. II. For the doubly bottom channels, where binding is already clearly established, the ultimate goal is to carry out simulations at not just the larger volume and single $m_{\pi}$ of the current study, but also, at this same volume, for multiple $m_{\pi}$, in order to provide updated results for the binding, extrapolated to physical $m_{\pi}$. The results of this extended update, which is in progress, will be reported elsewhere.

The rest of this paper is organized as follows. In Sec. II, we list the additional channels to be studied and review existing predictions for tetraquark binding in each. Section III provides a list of the operators used in these studies and Sec. IV a discussion of our gauge-fixed wall sources, and the extended, box-sink construction, introduced to improve our ground state signals. Section V provides details of our lattice setup and calculations, and Sec. VI the results of these calculations. Our final conclusions, together with a discussion of the implications of our results, are given in Sec. VII.

\section{CHANNELS OF INTEREST AND EXISTING TETRAQUARK PREDICTIONS THEREIN}

In this section, we specify the additional channels to be considered in this study, providing a review of existing tetraquark binding predictions for, and the reasons for an interest in each such choice. The predictions are to be tested against results from the lattice in Sec. VII.

The existence of sizeable $B_{c}$ data sets already establishes the existence of significant simultaneous production of $b \bar{b}$ and $c \bar{c}$ pairs. A bound tetraquark in one of the two mixedheavy charm-bottom channels is thus expected to be much more amenable to experimental detection than either of the $J^{P}=1^{+}, \overline{3}_{F}$ doubly bottom analogues discussed above. Replacing one of the two $\bar{b}$ antiquarks by a $\bar{c}$, however, is expected to both reduce the Coulomb attraction of the $3_{c}$ heavy antiquark pair and increase the residual spindependent heavy-light interactions, which heavy baryon spectrum splittings suggest is likely to further reduce binding. The bottom-charm states (if any) are thus qualitatively expected to be more weakly bound than their doubly bottom counterparts [9].

The mixed-heavy $I=0, J^{P}=1^{+} u d \bar{c} \bar{b}$ channel was investigated previously on the lattice [9]. Evidence of a 
possible bound ground state with binding between $\sim 15$ and $61 \mathrm{MeV}$ was found, though with rather short, late-time plateaus. The heavy quark symmetry arguments of Refs. [6,7], which produce binding compatible with that found from the lattice for the doubly bottom $I=0, J^{P}=1^{+}$ ground state, in contrast, predict no binding. A number of nonchiral models [17,19,20,34,37,42] also find an either unbound or only weakly bound ground state, with binding energies between $-1 \mathrm{MeV}$ (unbound) and $20 \mathrm{MeV}$ (depending on the details of the potential used) and $23 \mathrm{MeV}$ reported in Refs. [20,34], respectively. Significantly larger binding is found in most recent chiral model studies, where Refs. [36,38,41], for example, report ground states bound by $171 \pm 12 \mathrm{MeV}, 217 \mathrm{MeV}$ and $199 \mathrm{MeV}$, respectively. QCD sum rules, in contrast, produce inconclusive results, with binding of $60 \pm 100 \mathrm{MeV}$ reported in Ref. [45].

The $J^{P}=0^{+}, I=0, u d \bar{c} \bar{b}$ analogue has not yet been investigated on the lattice. The heavy quark symmetry arguments of Refs. [6,7] again predict no binding in this channel. Nonchiral models [18-20,33,34,37,42] predict an either unbound or only weakly bound ground state, with Refs. [20,33,34] quoting binding energies of between $-11 \mathrm{MeV}$ (unbound) and $13 \mathrm{MeV}$ (depending on the details of the potential used), $11 \pm 13 \mathrm{MeV}$, and $23 \mathrm{MeV}$, respectively. Significantly larger binding is found in most recent chiral model studies, with Refs. [36,38,41], for example, reporting bindings of $136 \pm 12 \mathrm{MeV}, 196 \mathrm{MeV}$ and $178 \mathrm{MeV}$, respectively. Two recent QCD sum rule studies differ in their pole mass results, with Ref. [45], which includes OPE contributions up to $D=8$, finding $7.14 \pm 0.10 \mathrm{GeV}$, while Ref. [48], which includes contributions up to $D=10$, finds $6660 \pm 150 \mathrm{MeV}$. The latter corresponds to a very deep, $485 \pm 150 \mathrm{MeV}$, binding relative to $D B$ threshold.

The $J^{P}=0^{+}$and $1^{+} \ell s \bar{c} \bar{b}$ channels have yet to be studied on the lattice. Analogous $J^{P}=1^{+}, \ell s \bar{b}^{\prime} \bar{b}$ states, with variable $\bar{b}^{\prime}$ mass as low as $\sim 0.6 m_{b}$, have, however, been considered, for $m_{\pi}=299 \mathrm{MeV}$ only, in Ref. [9]. With both $\bar{b}$ and $\bar{b}^{\prime}$ treated using nonrelativistic QCD (NRQCD), this study could not be extended to $\bar{b}^{\prime}$ masses as low as $m_{c}$; the observed variable $\bar{b}^{\prime}$ mass dependence, however, was argued to make a bound $\ell s \bar{c} \bar{b}$ state extremely unlikely in the $J^{P}=$ $1^{+}$channel at this pion mass. Both the heavy quark symmetry arguments of Refs. [6,7] and nonchiral model of Ref. [42] predict no tetraquark bound state in either this channel or the $J^{P}=0^{+}$analogue. In contrast, the QCD sum rule study of Ref. [54], which includes contributions up to $D=8$, finds binding energies of $200 \pm 130$ and $180 \pm$ $110 \mathrm{MeV}$ for the $J^{P}=0^{+}$and $1^{+}$ground states, respectively. Even deeper $J^{P}=0^{+}$bindings, exceeding $400 \mathrm{MeV}$, are reported in the QCD sum rule studies of Refs. [49,53], which include OPE contributions up to $D=10$.

The reported observation by the D0 Collaboration $[62,63]$ of a narrow state, $X(5568)$, decaying to $B_{s} \pi^{ \pm}$, and hence having four distinct flavors, prompted speculation that the related singly heavy $u d \bar{s} \bar{b}$ and $u d \bar{s} \bar{c}$ channels might support bound tetraquark states. The initial argument was based on the expectation that the putative $X(5568)$ should have a $U$-spin-interchanged $S U(3) u d \bar{s} \bar{b}$ partner with similar mass, coupled with the observation that the threshold, $5773 \mathrm{MeV}$, for the lowest-lying, two-meson $u d \bar{s} \bar{b}$ state, $B K$, lies well above the reported $X(5568)$ mass. While this initial motivation is weakened by the fact that searches by the LHCb [64], CMS [65], CDF [66] and ATLAS [67] Collaborations failed to confirm the D0 result, a number of model and QCD sum rule studies exist predicting bound tetraquark ground states in the $I=0$, $J^{P}=0^{+}$and $1^{+}, u d \bar{s} \bar{b}$ and/or $u d \bar{s} \bar{c}$ channels. The quark color delocalization screening model, for example, predicts $J^{P}=0^{+}$and $1^{+} u d \bar{s} \bar{b}$ ground states bound by 74 and $58 \mathrm{MeV}$ relative to the respective two-meson, $B K$ and $B^{*} K$, thresholds [39]. Another $S U(3)$ chiral quark model study, that of Ref. [35], obtains similar bindings, 70 and $68 \mathrm{MeV}$, for the $J^{P}=0^{+}$and $1^{+}$bottom-strange states. Somewhat smaller bindings, 19 and $16 \mathrm{MeV}$, respectively, are found in the alternate chiral quark model study of Ref. [41]. A bound tetraquark with even lower mass, $5380 \pm 170 \mathrm{MeV}$ (corresponding to a binding of $394 \pm 170 \mathrm{MeV}$ relative to $B K$ threshold), is also found for the $J^{P}=0^{+}$bottom-strange channel in the QCD sum rule study Ref. [51]. In fact, the only bottom-strange sector investigation we are aware of which does not produce a bound tetraquark state is the nonchiral-model, $I=0, J^{P}=1^{+}$channel study of Ref. [17]. This study also found no bound tetraquark in the $I=0$, $J^{P}=1^{+} u d \bar{s} \bar{c}$ channel. An absence of binding was also found for the related $I=0, J^{P}=0^{+} u d \bar{s} \bar{c}$ channel in the sum rule study of Ref. [51]. To our knowledge, the only other $u d \bar{s} \bar{c}$ channel study is that of Ref. [41], which finds $I=0, J^{P}=0^{+}$and $1^{+}$states bound by 15 and $9 \mathrm{MeV}$, respectively. Any bound state found in these channels would be of considerable phenomenological interest, since it would involve light degrees of freedom in the novel strangeness +1 , color $3_{c}$ configuration about which no phenomenological information is currently known.

While it might appear natural to include the $I=0, J^{P}=$ $1^{+} u d \bar{c} \bar{c}$ and $I=1 / 2, J^{P}=1^{+} \ell s \bar{c} \bar{c}$ channels in our study, we have not done so, since evidence already exists that bound doubly charmed tetraquark states, even if they exist in these channels, will be at most weakly bound. This evidence comes from both the lattice and the heavy-quarksymmetry arguments of Refs. [6,7], whose results are compatible with those of lattice studies for the doubly bottom bound states. The latter predicts no binding in either of the doubly charmed $J^{P}=1^{+}$channels. On the lattice, in the $I=0, J^{P}=1^{+} u d \bar{c} \bar{c}$ channel, (i) the results of Ref. [68] clearly establish the absence of binding at $m_{\pi}=391 \mathrm{MeV}$, (ii) Ref. [4] (which reaches a lower, $257 \mathrm{MeV}$, pion mass for the coarsest of the three lattices 
studied) finds an extrapolated, physical- $m_{\pi}$ binding of $23 \pm 11 \mathrm{MeV}$, sufficiently small for the authors to comment that further FV studies are required to reach a firm conclusion concerning binding, and (iii) results for the $b^{\prime}$ mass dependence of the binding energies for $I=0$, $J^{P}=1^{+} u d \bar{b}^{\prime} \bar{b}^{\prime}$ states at $m_{\pi}=299 \mathrm{MeV}$, reported in Ref. [9], strongly disfavor the possibility that binding survives to physical $m_{\pi}$ and $b^{\prime}$ masses as low as $m_{c}$. Only one lattice result, whose binding, $8 \pm 8 \mathrm{MeV}$, is compatible with a two-meson-threshold ground state, exists for the $I=1 / 2, J^{P}=1^{+} \ell s \bar{c} \bar{c}$ channel [4]. QCD sum rule studies also find no evidence for binding in either of the nonstrange $[43,44,46,55]$, or strange $[44,46,55]$ doubly charmed $J^{P}=1^{+}$channels. Nonchiral models whose $I=0, J^{P}=1^{+} u d \bar{b} \bar{b}$ bindings are compatible with those found on the lattice, similarly, predict either unbound $[17,22,27,29,33,36,37]$, or only weakly bound [24,26,30], doubly charmed $J^{P}=1^{+}$ground states. While many chiral quark model studies predict significant binding in the $I=0$, $J^{P}=1^{+} u d \bar{c} \bar{c}$ channel $[21,23,26,29,30,38,41]$, the models underlying these predictions typically also overbind the $I=0, J^{P}=1^{+}, u d \bar{b} \bar{b}$ ground state relative to results known from the lattice. We conclude that the absence of deeply bound tetraquark states in the two doubly charmed channels is already established and hence have not included these channels in the current study. Additional, larger volume ensembles would be required to reliably investigate such channels, where at-best-weak binding makes FV studies mandatory. In the future, we plan to generate additional ensembles with similarly low $m_{\pi}$, but larger volume than considered here, and will revisit the doubly charmed $J^{P}=1^{+}$channels when these become available.

Two final channels are studied in this paper. These are the triply heavy $J^{P}=1^{+} u c \bar{b} \bar{b}$ and $s c \bar{b} \bar{b}$ channels, considered previously only in the lattice study of Ref. [4], which found ground state masses compatible with the corresponding lowest two-meson thresholds. These channels are, of course, not among those where a bound tetraquark state, if it existed, would be more amenable to current experimental detection than the theoretically established doubly bottom states. They are included here only to provide an additional, independent check of the results of Ref. [4].

We close this section by stressing that deeply bound tetraquark ground states are predicted by some models (especially chiral quark models) and/or some QCD sum rule analyses in all of the $I=0, J^{P}=0^{+}$and $1^{+} u d \bar{c} \bar{b}, I=1 / 2$, $J^{P}=0^{+} \ell s \bar{c} \bar{b}$, and $I=0, J^{P}=0^{+}$and $1^{+} u d \bar{s} \bar{b}$ channels. Examples where predicted binding exceeds $100 \mathrm{MeV}$ can be found in (i) the recent sum rule studies of the $I=0, J^{P}=0^{+} u d \bar{c} \bar{b}, I=1 / 2, J^{P}=0^{+} \ell s \bar{c} \bar{b}$, and $I=0$, $J^{P}=0^{+} u d \bar{s} \bar{b}$ channels, which produce ground states $485 \pm$ $150 \mathrm{MeV}$ [48], $407 \pm 160 \mathrm{MeV}$ [49] or $467 \pm 150 \mathrm{MeV}$ [53], and $397 \pm 170 \mathrm{MeV}$ [52], respectively, below the corresponding lowest two-meson thresholds and (ii) recent chiral quark model studies of the $u d \bar{c} \bar{b}$ sector, which produce an $I=0, J^{P}=0^{+}$ground state $136 \pm 12 \mathrm{MeV}$ [36], $196 \mathrm{MeV}$ [38], or $178 \mathrm{MeV}$ [41] below $B D$ threshold and an $I=0, J^{P}=1^{+}$ground state $171 \pm 12 \mathrm{MeV}$ [36], $217 \mathrm{MeV}$ [38] or $199 \mathrm{MeV}$ [41] below $B^{*} D$ threshold. States bound this deeply should be readily amenable to detection in the multioperator lattice analyses detailed below.

It is also worth emphasizing that chiral and nonchiral quark models, all of which admit parametrizations which successfully reproduce the ordinary meson and baryon spectra, make qualitatively different predictions for the tetraquark channels discussed above. The nonchiral models, once one moves beyond the doubly bottom sector, predict only a few, usually at-most weakly bound tetraquark candidates. The majority of chiral model studies, in contrast, predict a larger number of much more deeply bound states. The lattice studies below should thus allow us to rule out at least one of these classes of models as providing an acceptable phenomenological representation of QCD in the low-energy regime.

\section{OPERATORS}

In this paper we work with the following set of operators having couplings to states with two quarks and two antiquarks with quark flavors $\psi, \phi, \theta$, and $\omega$, and having either "meson-meson" or "diquark-antidiquark" spin-colorflavor structure,

$$
\begin{aligned}
& D\left(\Gamma_{1}, \Gamma_{2}\right)=\left(\psi_{a}^{T} C \Gamma_{1} \phi_{b}\right)\left(\bar{\theta}_{a} C \Gamma_{2} \bar{\omega}_{b}^{T}\right), \\
& E\left(\Gamma_{1}, \Gamma_{2}\right)=\left(\psi_{a}^{T} C \Gamma_{1} \phi_{b}\right)\left(\bar{\theta}_{a} C \Gamma_{2} \bar{\omega}_{b}^{T}-\bar{\theta}_{b} C \Gamma_{2} \bar{\omega}_{a}^{T}\right), \\
& M\left(\Gamma_{1}, \Gamma_{2}\right)=\left(\bar{\theta} \Gamma_{1} \psi\right)\left(\bar{\omega} \Gamma_{2} \phi\right), \quad N\left(\Gamma_{1}, \Gamma_{2}\right)=\left(\bar{\theta} \Gamma_{1} \phi\right)\left(\bar{\omega} \Gamma_{2} \psi\right), \\
& O\left(\Gamma_{1}, \Gamma_{2}\right)=\left(\bar{\omega} \Gamma_{1} \psi\right)\left(\bar{\theta} \Gamma_{2} \phi\right), \quad P\left(\Gamma_{1}, \Gamma_{2}\right)=\left(\bar{\omega} \Gamma_{1} \phi\right)\left(\bar{\theta} \Gamma_{2} \psi\right) .
\end{aligned}
$$

The Dirac structures $\Gamma_{1}$ and $\Gamma_{2}$ relevant to the channels we consider are specified below. The short-hand terminologies "meson-meson" and "diquark-antidiquark" are meant only to compactly characterize the spin-color-flavor structures and should not be overinterpreted; there is nothing, for example, to prevent one of the "meson-meson" operators from coupling to a state with a spatially extended diquarkantidiquark structure or one of the "diquark-antidiquark" operators from coupling to a meson-meson scattering state. An illustration is provided by the deeply bound ground state in the doubly bottom, $I=0, J^{P}=1^{+}$channel, which was found to couple strongly to both the "meson-meson" and "diquark-antidiquark" operators considered in Ref. [3].

The lattice explicitly breaks rotational symmetry, breaking the continuum rotation group down to the little group $O_{h}$. The operators listed above lie in either the $A_{1}$ or $T_{1}$ irrep, which map directly to the continuum $J=0$ and 
TABLE I. $J^{P}=1^{+}$operators used in this work.

\begin{tabular}{|c|c|c|c|}
\hline Type $(\psi \phi \theta \omega)$ & $I(J)^{P}$ & Diquark-antidiquark & Dimeson \\
\hline \multirow[t]{3}{*}{$u d c b / u d s b / u d s c$} & \multirow{3}{*}{$0(1)^{+}$} & $D\left(\gamma_{5}, \gamma_{i}\right), D\left(\gamma_{t} \gamma_{5}, \gamma_{i} \gamma_{t}\right)$ & $\begin{array}{c}M\left(\gamma_{5}, \gamma_{i}\right)-N\left(\gamma_{5}, \gamma_{i}\right) \\
M\left(I, \gamma_{i} \gamma_{5}\right)-N\left(I, \gamma_{i} \gamma_{5}\right)\end{array}$ \\
\hline & & & $O\left(\gamma_{5}, \gamma_{i}\right)-P\left(\gamma_{5}, \gamma_{i}\right)$ \\
\hline & & $E\left(\gamma_{5}, \gamma_{i}\right), E\left(\gamma_{t} \gamma_{5}, \gamma_{i} \gamma_{t}\right)$ & $\begin{array}{c}O\left(I, \gamma_{i} \gamma_{5}\right)-P\left(I, \gamma_{i} \gamma_{5}\right) \\
\epsilon_{i j k} M\left(\gamma_{j}, \gamma_{k}\right)\end{array}$ \\
\hline$u d b b$ & \multirow[t]{2}{*}{$0(1)^{+}$} & \multirow[t]{2}{*}{$D\left(\gamma_{5}, \gamma_{i}\right), D\left(\gamma_{t} \gamma_{5}, \gamma_{i} \gamma_{t}\right)$} & $\begin{array}{c}M\left(\gamma_{5}, \gamma_{i}\right)-N\left(\gamma_{5}, \gamma_{i}\right) \\
M\left(I, \gamma_{i} \gamma_{5}\right)-N\left(I, \gamma_{i} \gamma_{5}\right)\end{array}$ \\
\hline \multirow[t]{2}{*}{$l s b b / u c b b / s c b b$} & & & $M\left(\gamma_{5}, \gamma_{i}\right), M\left(I, \gamma_{i} \gamma_{5}\right)$ \\
\hline & $\frac{1}{2}(1)^{+}$ & $D\left(\gamma_{5}, \gamma_{i}\right), D\left(\gamma_{t} \gamma_{5}, \gamma_{i} \gamma_{t}\right)$ & $\begin{array}{c}N\left(\gamma_{5}, \gamma_{i}\right), N\left(I, \gamma_{i} \gamma_{5}\right) \\
\epsilon_{i j k} M\left(\gamma_{j}, \gamma_{k}\right)\end{array}$ \\
\hline \multirow[t]{3}{*}{$u s c b$} & \multirow{3}{*}{$\frac{1}{2}(1)^{+}$} & $D\left(\gamma_{5}, \gamma_{i}\right), D\left(\gamma_{t} \gamma_{5}, \gamma_{i} \gamma_{t}\right)$ & $M\left(\gamma_{5}, \gamma_{i}\right), M\left(I, \gamma_{i} \gamma_{5}\right)$ \\
\hline & & & $N\left(\gamma_{5}, \gamma_{i}\right), N\left(I, \gamma_{i} \gamma_{5}\right)$ \\
\hline & & $E\left(\gamma_{5}, \gamma_{i}\right), E\left(\gamma_{t} \gamma_{5}, \gamma_{i} \gamma_{t}\right)$ & $\begin{array}{c}O\left(\gamma_{5}, \gamma_{i}\right), O\left(I, \gamma_{i} \gamma_{5}\right) \\
\epsilon_{i j k} M\left(\gamma_{j}, \gamma_{k}\right)\end{array}$ \\
\hline
\end{tabular}

$J=1$ quantum numbers respectively. For the operators that are produced from the product $T_{1} \otimes T_{1}=A_{1} \otimes E \otimes$ $T_{1} \otimes T_{2}$ [69] we pick out the scalar and vector components respectively [5].

The operator combinations which in principle couple to states in the $J^{P}=0^{+}$and $1^{+}$channels considered below are listed in Tables II and I, respectively. While the diquark-antidiquark operators, $D$ and $E$, were included in initial explorations of the $u d \bar{s} \bar{c}$ and $u d \bar{s} \bar{b}$ channels, they were found to couple only weakly to the corresponding ground states and hence not included in the final analyses of these channels. They were also omitted from the final version of the analyses of the $u d \bar{c} \bar{b}$ channels where using the set of meson-meson operators was found to give more statistically precise results and produce no significant change to the energies of the lowest-lying states.

Throughout this work we will obtain our ground states from a generalized eigenvalue problem (GEVP) approach [70-72]. We use the solutions of the GEVP (sample by sample) to extract the "optimized correlator,"

$$
C_{i}(t)=\sum_{j, k} V_{i j}(\tau)^{\dagger} C_{j k}(t) V_{k i}(\tau),
$$

where $V$ is the matrix with columns made of the eigenvector solutions of

$$
C_{i j}(t) v_{j}(t)=\lambda_{i} C_{i j}\left(t+t_{0}\right) v_{j}(t)
$$

$\tau$ (the "diagonalization time" [73]) in Eq. (2) is to be chosen large enough to produce improved projection onto the ground state. We then perform a correlated singleexponential fit to the resulting optimized correlators to obtain our final levels. In the rest of this work we will quote results obtained using $t_{0} / a=2$ and $\tau / a=4$, values for which the ground state masses are found to display good stability upon variation of these parameters. Larger values of $t_{0}$ and $\tau$ tend to make the solution statistically less precise and eventually unstable.

\section{WALL SOURCES AND THE BOX-SINK CONSTRUCTION}

Throughout this work we will use Coulomb-gauge-fixed wall sources [74,75] (fixed to high-precision using the Fourier-accelerated conjugate gradient algorithm of [76]). These sources have some benefits, as well as some peculiarities, which are discussed below.

A gauge-fixed wall source is a time slice of point sources on a Coulomb gauge-fixed background. Here we assume the gauge condition has been applied over the whole volume. The elements of the source do not need to be stochastically drawn as the gauge fixing condition connects colors appropriately; this means that these sources can be

TABLE II. $\quad J^{P}=0^{+}$operators used in this work.

\begin{tabular}{llcc}
\hline \hline Type $(\psi \phi \theta \omega)$ & $I(J)^{P}$ & Diquark-antidiquark & Dimeson \\
\hline udcb/udsb/udsc & $0(0)^{+}$ & $E\left(\gamma_{5}, \gamma_{5}\right), E\left(\gamma_{t} \gamma_{5}, \gamma_{t} \gamma_{5}\right)$ & $M\left(\gamma_{5}, \gamma_{5}\right)-N\left(\gamma_{5}, \gamma_{5}\right)$ \\
& & $M(I, I)-N(I, I)$ \\
uscb & & $M\left(\gamma_{i}, \gamma_{i}\right)$ \\
& $\frac{1}{2}(0)^{+}$ & $E\left(\gamma_{5}, \gamma_{5}\right), E\left(\gamma_{t} \gamma_{5}, \gamma_{t} \gamma_{5}\right)$ & $M\left(\gamma_{5}, \gamma_{5}\right), M(I, I)$ \\
& & $N\left(\gamma_{5}, \gamma_{5}\right), N(I, I)$ \\
& & $M\left(\gamma_{i}, \gamma_{i}\right)$ \\
\hline \hline
\end{tabular}


used for baryons [77-79]. Gauge-fixed wall source results can be considerably more statistically precise, especially at lighter pion masses, than point sources at the same cost due to the volume-sampling. Like other wall sources, these automatically zero-momentum project, and if momentum is needed it must be put into the source explicitly as a momentum source or introduced via partial twisting [80,81], as was done for the NRQCD tuning (see Appendix A).

Use of a gauge-fixed wall source is much like that of a point source, though with some potential complications for channels like those studied here where two-meson thresholds exist. When combined with a local sink, one typically finds a negative sign for the amplitude of (at least) the first excited state and hence a "wall-local" correlator whose effective mass approaches the ground state mass from below. If one cannot measure the signal to large enough $t$ to ensure the ground state plateau has been reached, the ground state mass may then be underestimated and lead one to either conclude that a bound state exists when in fact one does not or overestimate the binding in the case one does exist. This issue does not arise for point or stochastic wall sources, where the effective masses approach the groundstate mass from above and so offer a rigorous upper bound on that mass.

Often it useful to compute "wall-wall" correlators [82], defined by contracting objects in the usual way, with propagators that are summed over the spatial sites of the sink time slice,

$$
S^{W}(t)=\sum_{x} S(x, t)
$$

The resulting "wall-wall" correlation functions are symmetric under the exchange of operators at the source and sink and have effective masses which approach the ground state from above. They are, however, very

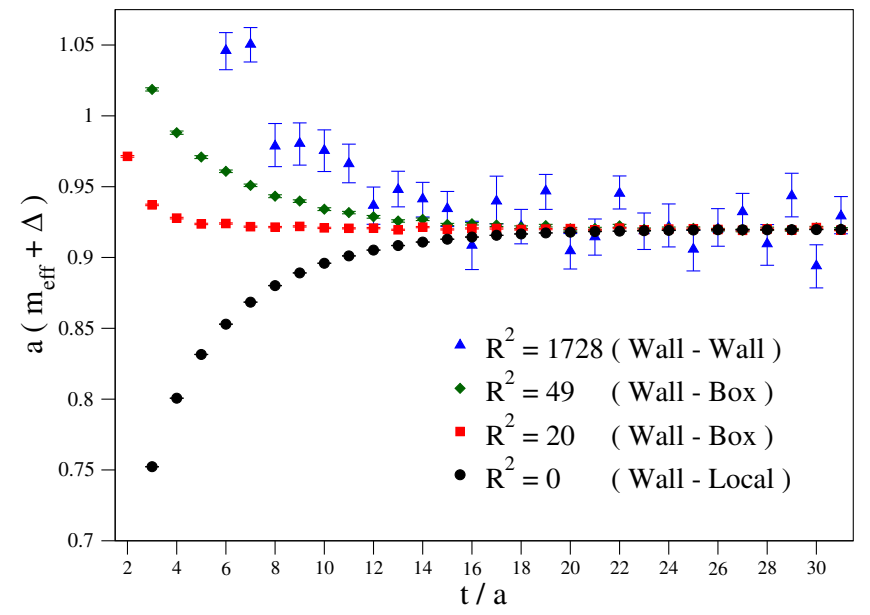

statistically noisy. Since wall-local correlator effective masses empirically approach their ground state plateaus from below (i.e., have negative excited state contamination) while those of wall-wall correlators approach theirs from above (i.e., have positive excited state contamination), sink combinations intermediate between the two are expected to exist with small excited state contamination. The goal is to find, if possible, combinations of this type which also suffer from only a limited loss in statistical precision. One possibility would be to use some form of sink smearing, though the products of link matrices needed with the propagator solution would make that computationally costly. Another would be to simply take advantage of the gauge condition and construct propagators by summing over points lying inside a sphere around each reference sink point $x$,

$$
S^{B}(x, t)=\frac{1}{N} \sum_{r^{2} \leq R^{2}} S(x+r, t) .
$$

With this construction, varying $R^{2}$ between 0 and its maximum value, $3(L / 2)^{2}$, produces a continuous interpolation between the wall-local and wall-wall cases. We refer to sinks constructed in this manner as "box-sinks." As the boxsink sum can be performed at the level of contractions and does not require extra inversions, the construction of wallbox correlators is reasonably cheap to implement. In channels where the ground state is a compact hadronic object, one would expect an optimal wall-box improvement of the ground state plateau for box-sink radii roughly matching the physical ground-state hadron size.

One should bear in mind that, in contrast to what happens in the wall-wall case, wall-box and wall-local correlation matrices are not symmetric [83]. The GEVP method, however, does not necessarily need this symmetry to obtain the underlying real eigenvalues. Careful monitoring of the imaginary parts of the eigenvalues is needed, and in practice the lack of symmetry was not found to be an issue.

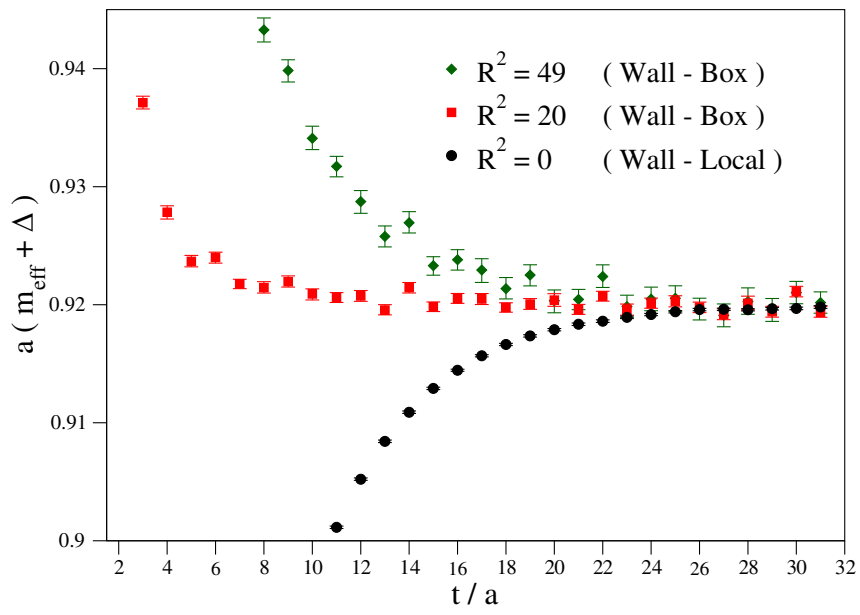

FIG. 1. An illustrative comparison of box-sink $B_{c}$ meson correlation function results for different box-sink radii. $R^{2}$ is in lattice units. The right panel shows a zoom into the plateau region. 


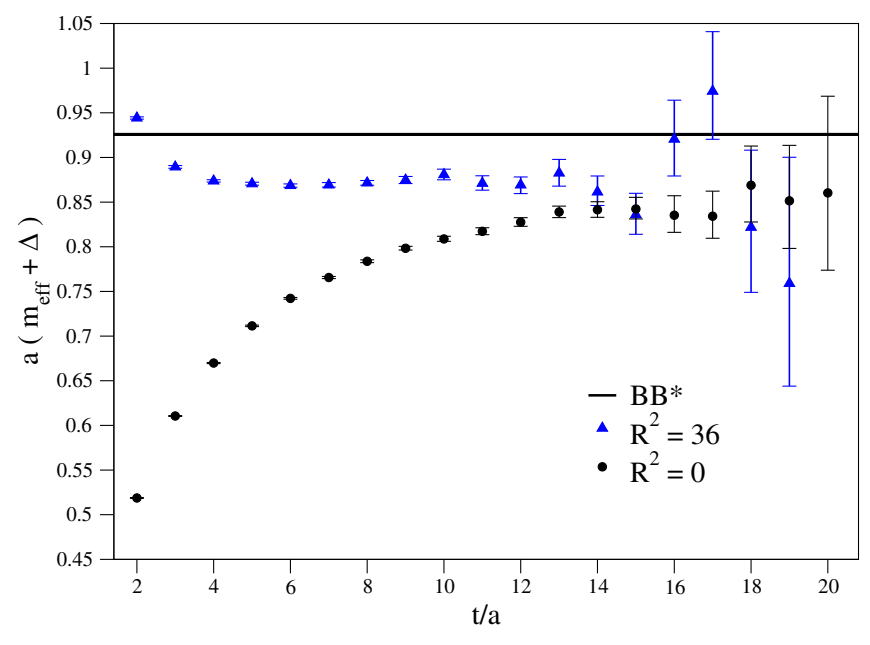

(a) $u d \bar{b} \bar{b}$

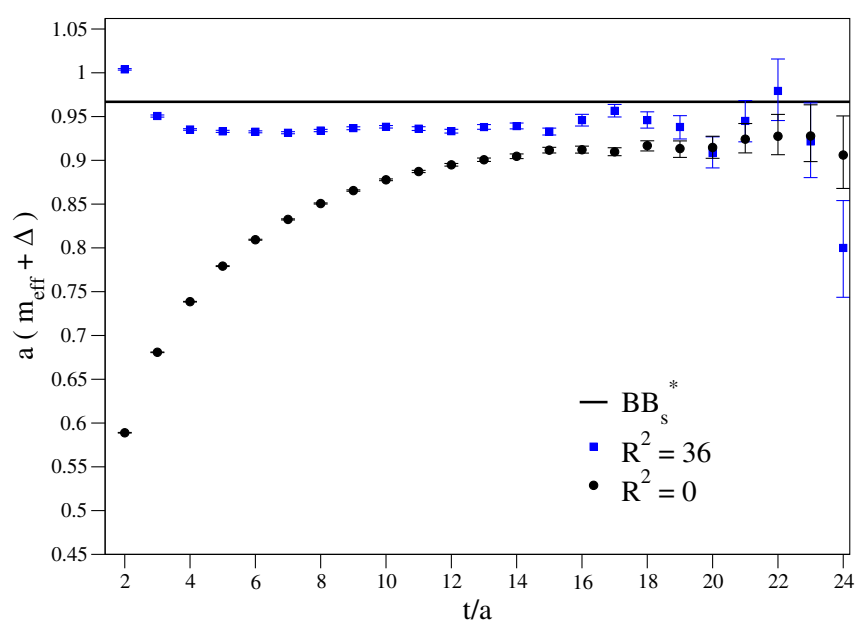

(b) $\ell s \bar{b} \bar{b}$

FIG. 2. A comparison of the wall-local and optimal- $R^{2}$ box-sink results for the $u d \bar{b} \bar{b}$ and $l s \bar{b} \bar{b}$ channel ground states.

An illustration of the utility of the box-sink construction is provided, for the case of the $B_{c}$ meson channel, in Fig. 1. We see that intermediate values of $R^{2}$, indeed, exist which extend the ground state plateau to significantly lower $t$ than is the case for the wall-local or wall-wall combinations. Results for all box-sink radii must, of course, approach the same ground state mass at sufficiently large $t$. The point here is that the improvement in the ground state plateau occurs for small enough $R^{2}$ that the onset of the significant increase in noise that occurs as $R^{2}$ grows towards the wallwall limit is avoided in the optimal $R^{2}$ plateau region. The figure also illustrates that a simple wall-local correlator can have significant excited state contamination and only approaches the plateau at very large times. This is less of a problem in single-meson channels, like the $B_{c}$ channel,

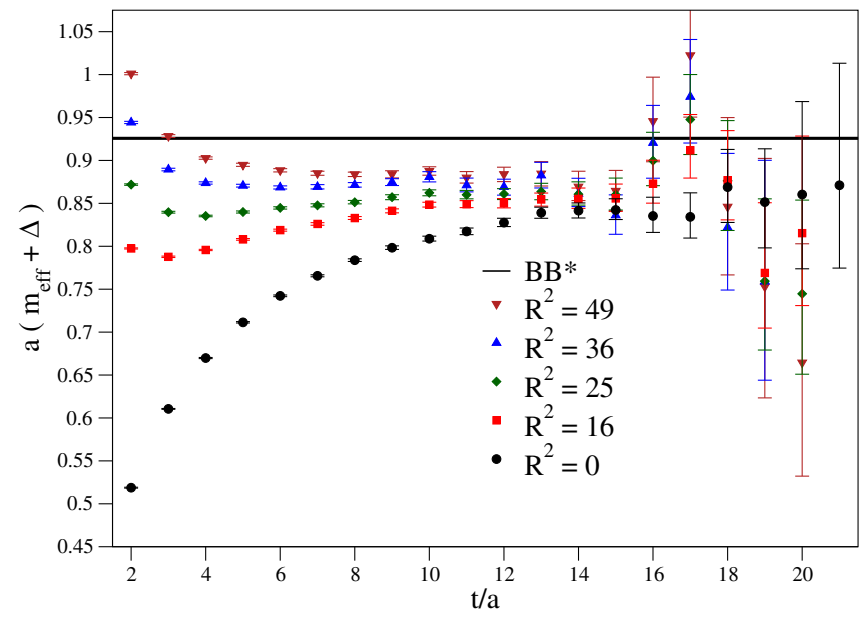

(a) $u d \bar{b} \bar{b}$ where one can follow the signal out to large enough $t$, but becomes an issue in channels with larger signal-to-noise problems, such as the tetraquark channels we study here, where we are typically only able to follow our signal out to $t / a \approx 20$ due to an exponential signal to noise problem. Such issues have been discussed in the literature for a long time in the context of the nucleon and $\rho$ meson; see, e.g., Refs. [77,78,84].

A further illustration of the utility of the box-sink construction, now for tetraquark signals, is provided by effective mass plots of Figs. 2 and 3, which display optimized ground-state correlator results for the $J^{P}=1^{+}, I=0 u d \bar{b} \bar{b}$ and $I=1 / 2 \ell s \bar{b} \bar{b}$ channels. Figure 2 provides a comparison of the wall-local and corresponding optimized $\left(R_{\mathrm{opt}}^{2}=36\right)$ box-sink results. While at fixed $t / a$, the smearing inherent in

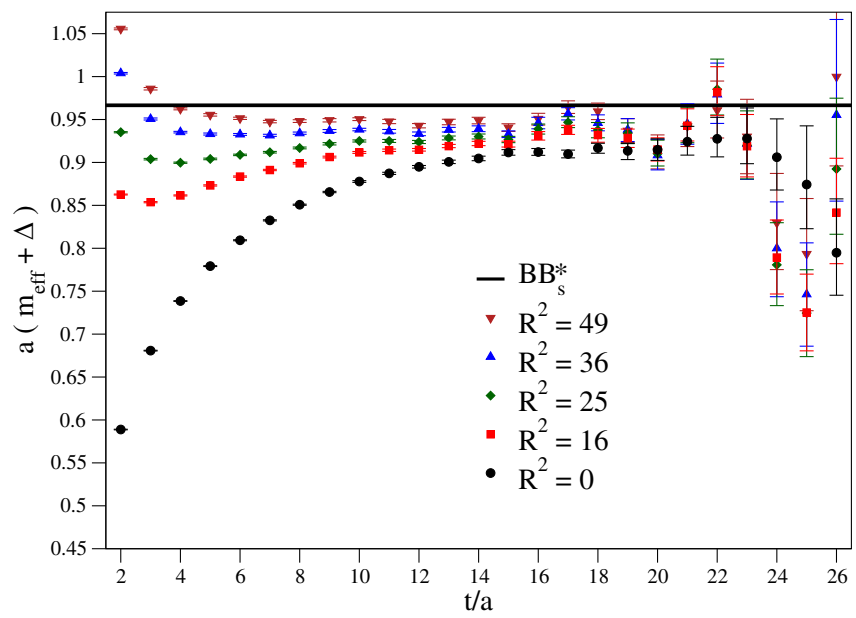

(b) $\ell s \bar{b} \bar{b}$

FIG. 3. Box-sink effective mass plots for the lowest GEVP state in the $J^{P}=1^{+}, I=0 u d \bar{b} \bar{b}$ and $I=1 / 2, \ell s \bar{b} \bar{b}$ channels. Also shown is the lowest-lying two-meson threshold at rest. 
TABLE III. Values of the box-sink summation radii (in lattice units) used in this work. Values were chosen so the corresponding effective mass plateaus begin reasonably early.

\begin{tabular}{lrrrrrr}
\hline \hline Operators & Flavor & $R^{2}$ & Flavor & $R^{2}$ & Flavor & $R^{2}$ \\
\hline Single mesons & $\bar{u} d$ & 0 & $\bar{s} u$ & 0 & $\bar{c} u$ & 49 \\
& $\bar{c} s$ & 49 & $\bar{b} u$ & 49 & $\bar{b} s$ & 49 \\
& $\bar{b} c$ & 20 & & & & \\
Tetraquarks & $u d \bar{s} \bar{c}$ & 49 & $u d \bar{s} \bar{b}$ & 49 & $u d \bar{c} \bar{b}$ & 49 \\
& $u s \bar{c} \bar{b}$ & 64 & $u c \bar{b} \bar{b}$ & 36 & $s c \bar{b} \bar{b}$ & 36 \\
\hline \hline
\end{tabular}

the box-sink construction produces optimized box-sink errors larger than those on the corresponding wall-local signal at the same $t / a$, the box-sink signal, in both channels, plateaus at much earlier times, at ground-state effective masses compatible with those of the corresponding late-time wall-local results. Crucially, the errors in these early box-sink plateau regions are, as in the $B_{c}$ case, significantly smaller than those in the regions of the corresponding shorter, latertime wall-local plateaus. This advantage is especially important for the tetraquark channels, where, in comparison to the $B_{c}$ case, the wall-local signal degrades at significantly earlier $t / a$. This loss of signal at earlier $t / a$, combined with the later onset of the wall-local plateaus, leads to rather short, latetime plateaus, and larger-error wall-local tetraquark results. Figure 3 provides additional information on the impact of the box-sink construction for bound tetraquark signals, displaying results for the ground-state effective masses of the two doubly bottom tetraquark channels, now for a range of boxsink radii. The results show a steady growth in the errors at fixed- $t / a$ and a loss of signal to noise at increasingly early $t / a$ as $R^{2}$ is increased from the wall-local to the wall-wall limit. For $R^{2}<R_{\mathrm{opt}}^{2}$, the ground-state signal approaches the plateau from below, signaling negative excited-state contamination, while for $R^{2}>R_{\mathrm{opt}}^{2}$, it approaches from above, signaling positive excited-state contamination. The optimized choice $\left(R_{\mathrm{opt}}^{2}=36\right.$ for these channels), appears, as expected, to successfully minimize excited-state contamination at earlier times. It also, as expected for channels having bound tetraquark ground states, corresponds to a physical smearing radius of typical hadronic size, the radii used in this work are listed in Table III.

The fact that enhanced excited-state contamination is seen in the wall-local results of both the $B_{c}$ and tetraquark channels suggests this behavior may be common for walllocal correlators. Figure 3 illustrates, in more detail, the potential danger this creates. In the absence of the longer, more reliable box-sink plateaus, it would be easy, with only the wall-local results, to mistakenly identify the start of the wall-local plateaus as occurring around $t / a=14$, leading to an overestimate of the binding in both channels. The utility of the box-sink construction in avoiding this problem is clear. Given the results shown in Figs. 2 and 3, we expect our earlier wall-local-correlator-based estimates for the physical point bindings in these channels [3] to be reduced once the ongoing improved, multiple- $m_{\pi}$ box-sink analysis is completed. For now, the results for these channels, at the single $m_{\pi}$ of the current study, serve to establish the boxsink construction as an improved method for identifying channels supporting deeply bound tetraquark states and quantifying the binding therein, and motivate our use of it in the studies of the other channels, reported below.

\section{LATTICE DETAILS}

For all channels investigated in this work we have used a single $n_{f}=2+1,48^{3} \times 64$ Wilson-Clover ensemble generated as an extension to the PACS-CS ensembles [85] used in our previous works $[3,9]$. As this ensemble is generated with the same $\beta$ and $C_{S W}$, the lattice-spacing [for which we use $a^{-1}=2.194(10) \mathrm{GeV}$ [86] ] and the charm-quark action parameters should be the same as for those previous ensembles.

This ensemble was generated using openQCD-1.6 [87], and all propagator inversions were performed using a version of this code (modified [88]) for the charm quarks as well. For the light and strange quarks we use the DFL + SAP + GCR solver algorithm [89-91] and solve for multiple wall source positions as the same deflation subspace can be used. For the charm quarks, we just use the SAP + GCR solver as low-mode deflation is practically ineffective for very heavy quarks and there is a significant overhead in generating the deflation subspace compared to the solve time. For the charm-quark action we use the relativistic heavy quark action of Refs. [92,93] with the same parameters as listed in [94]. For the b-quark propagators we use tadpole-improved, tree-level NRQCD propagators generated on-the-fly from the gauge configuration. With several technical improvements to our generation of NRQCD propagators the combination of partially twisted boundary conditions and the beneficial statistical properties of gauge-fixed wall sources could be used to tune our bare-mass parameter to significantly higher precision than before. The partially twisted boundary conditions, in particular, allow for a denser selection of momenta in the $\eta_{b}$ and $\Upsilon$ dispersion relations within a lattice irrep than is possible with only Fourier modes and thus improve the statistical resolution. Details of our action and of the new tuning procedure we have used are provided in Appendix A. Almost $1000 \mathrm{u}, \mathrm{s}, \mathrm{c}$ and b propagators have been generated for each of the tetraquark channels considered here.

The new ensemble has some attractive features. In particular, its pion mass, $\simeq 192 \mathrm{MeV}$, is reasonably close to physical while still maintaining $m_{\pi} L>4$. As for the previous PACS-CS ensembles, we have a strange sea quark heavier than physical and thus have to use a partially quenched strange (using the $\kappa_{s}$-value of [95]) to get a respectable $(507 \mathrm{MeV})$ kaon mass. The Tsukuba tuning also gives close to physical $D$ and $D^{*}$ meson masses. The lattice-valued masses we obtain are presented in Table VI 
TABLE IV. Details of the ensemble used in this work.

\begin{tabular}{lcccccc}
\hline \hline$V$ & $\kappa_{l}$ & $\kappa_{s}^{\text {sea }}$ & $\kappa_{s}^{P Q}$ & $N_{\text {conf }}$ & $N_{\text {src }}$ & $m_{\pi} L$ \\
\hline $48^{3} \times 64$ & 0.13777 & 0.13640 & 0.13666 & 122 & 8 & 4.2 \\
\hline \hline
\end{tabular}

in Appendix C. Note that, for NRQCD, where there is an additive mass renormalization, $\Delta$, only mass differences have physical meaning. We will present results in this lattice-valued form for the majority of this work.

As we use NRQCD for the $b$ quarks, channels with one or more $b$ quarks do not have a rigorous continuum limit. We do, however, include terms that should cancel up to $O\left(a^{4}\right)$ discretization effects in our NRQCD Hamiltonian [96]. The c quarks have been tuned to have a flat dispersion relation and so are expected to have a very mild approach to the continuum. The light-quarks are $O(a)$-improved nonperturbatively with the $C_{S W}$ term. In all, we expect discretization effects to be relatively small.

Table IV provides a summary of the new ensemble parameters, the number of configurations, $N_{\text {conf }}$, and the number of sources per configuration, $N_{\text {src }}$, used in this study.

All vector correlators were averaged over the spatial index $i$, and all correlators were symmetrized with their backwardpropagating states in order to improve statistical resolution.

\section{RESULTS}

In this section, we present the measured spectra (GEVP eigenvalues) and associated two-meson thresholds for the channels discussed above. The eigenvalues are obtained from correlated, single-exponential fits to the corresponding optimised correlators. The windows of $t$ used in these fits are tabulated in Appendix $\mathrm{C}$ and the corresponding effective-mass plots, showing results for the ground states, as well as those excited states for which some signal could be resolved, collected in Appendix D. Reliable, early-onset

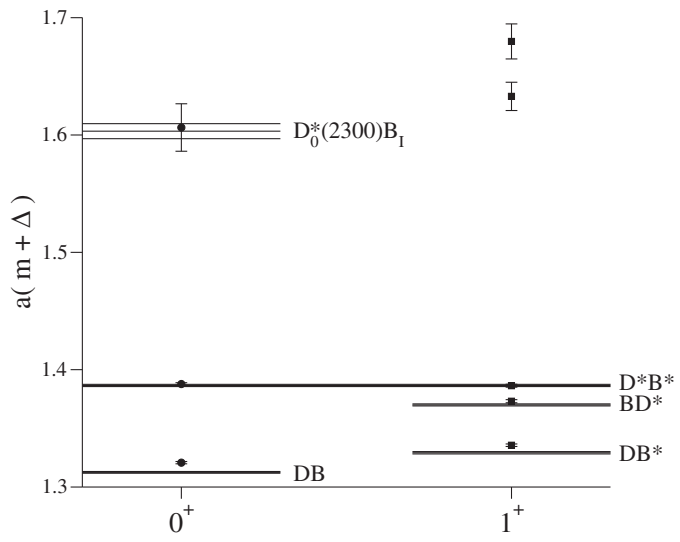

(a) $u d \bar{c} \bar{b}$ ground-state plateaus are evident in all of the channels considered. Additional low-lying excited states are also typically well determined. Results for a number of higherlying, less well determined excited states, obtained from fits to signals lost at relatively early times and/or with relatively short fit windows are also included in the tables and spectrum plots. These serve to identify, qualitatively, the parts of higher-lying two-meson spectrum which couple most strongly to the operators under consideration.

Figure 4(a) shows the spectrum results for the $I=0$, $J^{P}=0^{+}$and $1^{+} u d \bar{c} \bar{b}$ channels. The ground states in both cases are found to be roughly consistent with the lowest two-meson threshold, with no further states near threshold. There is thus no sign of an extra, bound tetraquark state in either channel.

The results for the $J^{P}=0^{+}$and $1^{+} \ell s \bar{c} \bar{b}$ channels are shown in Fig. 4(b). Here we see the ground states in both channels pushed marginally below the corresponding lowest two-meson thresholds, $D B_{s}$ and $D B_{s}^{*}$, respectively. With no other states in the vicinity, however, we suspect this reflects either a residual systematic (from either the GEVP procedure or our fitting to the optimised groundstate correlators) or a small $(<10 \mathrm{MeV}) \mathrm{FV}$ shift and that the ground states in these channels correspond to the lowest two-meson thresholds. With only a single volume, the possibility of weakly bound ground states cannot, however, be unambiguously ruled out. Interestingly, the three highest excited states in the $J^{P}=1^{+}$channel all lie just below higher-lying two-meson thresholds, with splittings consistent with those between these thresholds. This "pushing down" of the eigenvalues is of the order of a few $\mathrm{MeV}$ at most. This pattern is not, however, observed for the $J^{P}=0^{+}$channel, where the third excited state lies much higher than the third excited two-meson threshold, $D^{*} B_{s}^{*}$.

Figure 5(a) shows our results for the $I=0, J^{P}=0^{+}$ and $1^{+} u d \bar{s} \bar{b}$ channels. The ground states again come out

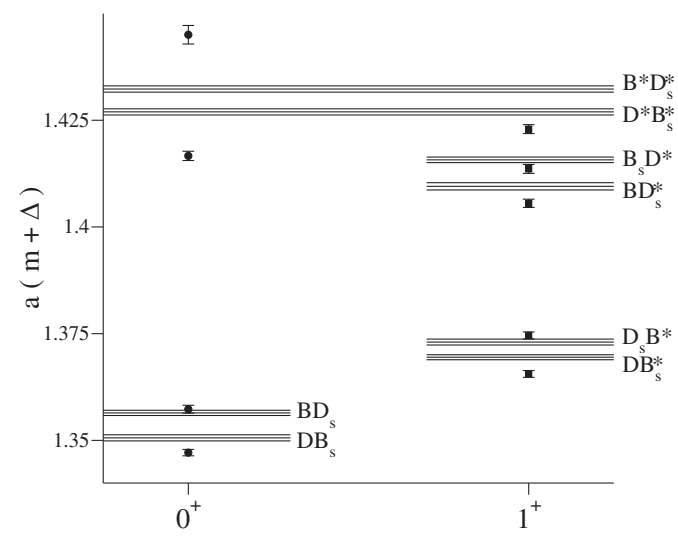

(b) $\ell s \bar{c} \bar{b}$

FIG. 4. Lowest-lying GEVP eigenvalues and associated two-meson thresholds for the $I=0, J^{P}=0^{+}$and $1^{+} u d \bar{c} \bar{b}$ (left) and $J^{P}=0^{+}$ and $1^{+} \ell s \bar{c} \bar{b}$ (right) channels. 


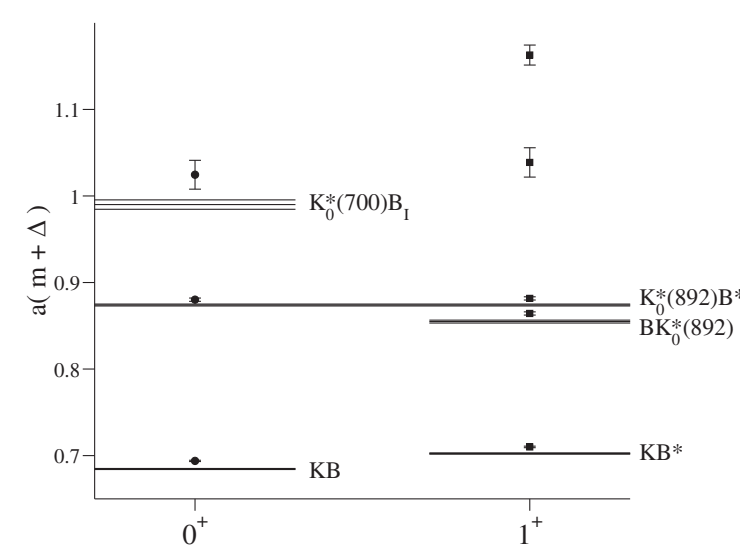

(a) $u d \bar{s} \bar{b}$

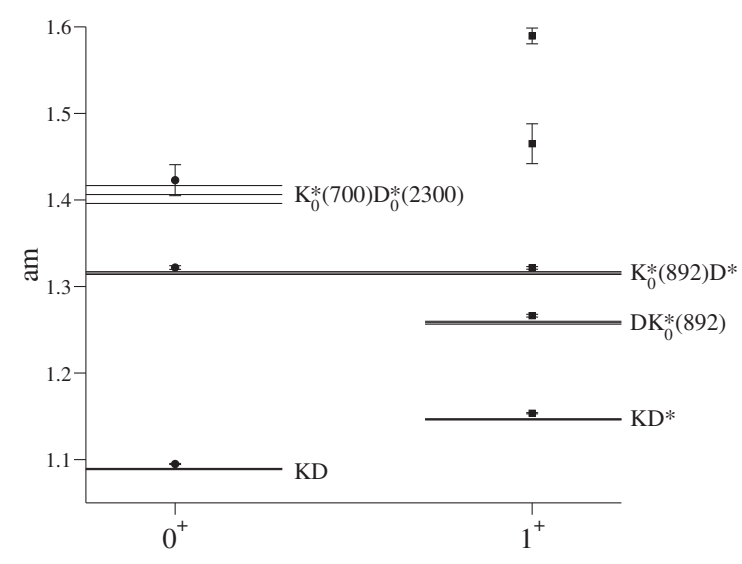

(b) $u d \bar{s} \bar{c}$

FIG. 5. Lowest-lying GEVP eigenvalues and associated two-meson thresholds for the $I=0, J^{P}=0^{+}$and $1^{+} u d \bar{s} \bar{b}$ (left) and $u d \bar{s} \bar{c}$ (right) channels.

consistent with the corresponding lowest two-meson thresholds, with no sign of an extra state below, or even near, these thresholds, suggesting the observed ground states once more correspond to the two-meson threshold states. Results for the analogous $I=0, J^{P}=0^{+}$and $1^{+} u d \bar{s} \bar{c}$ channels are shown in Fig. 5(b). The ground states lie slightly above the lowest two-meson thresholds, with, once more, no sign of an additional, bound state below threshold in either channel.

Finally, in Fig. 6 we show our results for the triply heavy $J^{P}=1^{+}, u c \bar{b} \bar{b}$ and $s c \bar{b} \bar{b}$ channels. It appears there is a state in both these channels with energy shifted from that of the expected two-meson threshold with both mesons at rest. It is possible this corresponds to a scattering state or even a resonance. A more precise understanding of the levelstructure in these channels would require a dedicated finitevolume study with the inclusion of meson-meson operators with nonzero back-to-back momentum, which is beyond the scope of the present work. The ground states are again

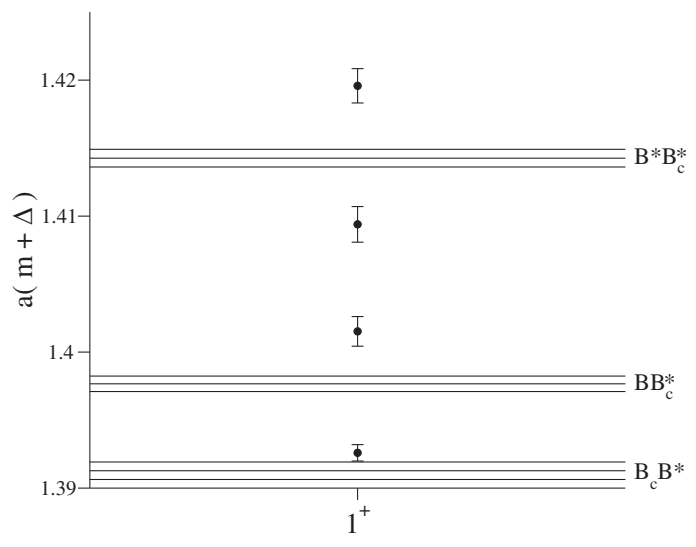

(a) $u c \bar{b} \bar{b}$ consistent with the corresponding two-meson thresholds $\left(B B_{c}^{*}\right.$ and $B_{s} B_{c}^{*}$, respectively) and are in reasonable agreement with the unbound or very shallowly bound results for these states reported in Ref. [4].

The lowest-lying resolvable states can be found in Tables IX, X, XI, XII in Appendix C.

\section{DISCUSSION AND CONCLUSIONS}

In this work we have used lattice simulations to investigate a number of exotic tetraquark channels in which model and/or QCD sum rule studies and/or heavy-quark symmetry arguments predict bound, strong-interactionstable tetraquark states to exist. We have also revisited our earlier lattice studies of the doubly bottom, $J^{P}=1^{+}, \overline{3}_{F}$ $u d \bar{b} \bar{b}$ and $\ell s \bar{b} \bar{b}$ and bottom-charm, $J^{P}=1^{+}, I=0 u d \bar{c} \bar{b}$ channels [3,9], introducing a number of significant improvements over those earlier works. In particular, we have (i) generated a new ensemble with close-to-physical

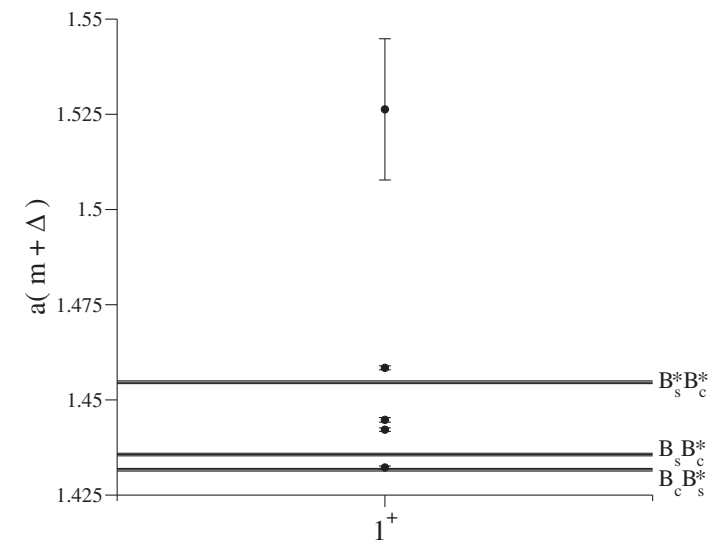

(b) $s c \bar{b} \bar{b}$

FIG. 6. Lowest-lying GEVP eigenvalues and associated two-meson thresholds for the $J^{P}=1^{+} u c \bar{b} \bar{b}$ and $s c \bar{b} \bar{b}$ channels. 
$(\simeq 192 \mathrm{MeV})$ pion mass and significantly larger volume $\left(m_{\pi} L=4.2>4\right)$ to better control possible finite volume effects; (ii) added additional operators to improve our ability to resolve the tower of states contributing to each correlator, and (iii) made major improvements to our ground-state signals via the box-sink construction. We have also, as described in more detail in Appendix A, retuned our NRQCD bare mass to a much more precise and reliable value, using partially twisted boundary conditions. All of these represent dramatic improvements over our previous work.

The only channels in which we find evidence for deeply bound, strong-interaction-stable tetraquark states are the doubly bottom $J^{P}=1^{+}, \overline{3}_{F}, u d \bar{b} \bar{b}$ and $\ell s \bar{b} \bar{b}$ channels already identified as supporting such bound states in earlier lattice studies, including our own. Our new results for these channels, albeit so far only at a single $m_{\pi} \simeq 192 \mathrm{MeV}$, display significantly improved ground-state plateaus, largely due to the improved, box-sink construction. With the bindings at this $m_{\pi}$ reduced compared to those implied for the same $m_{\pi}$ by the fits to the $m_{\pi}$ dependences reported in Ref. [3], we expect to find similarly reduced physical- $m_{\pi}$ results once we complete our ongoing multiple- $m_{\pi}$ analysis of these channels and are able to perform the physical-point extrapolation. With the binding in these channels expected to increase with decreasing $m_{\pi}$, the final physical-point bindings will be deeper than those of the current study. ${ }^{1}$ The current, single- $m_{\pi}$ results, thus provide further confirmation of the conclusions reached in previous lattice studies regarding the existence of a $\overline{3}_{F}$ of stronginteraction-stable, $J^{P}=1^{+}$, doubly bottom tetraquark states. Once the multiple- $m_{\pi}$ analysis has been completed, the improved ground state signals produced by the box-sink construction should also significantly improve the reliability of the extrapolated physical $-m_{\pi}$ results.

In contrast to the doubly bottom, $\overline{3}_{F}, J^{P}=1^{+}$channels, no bound, nonmolecular ground states are found in any of the other ten channels considered here. States with bindings $\gtrsim 10-20 \mathrm{MeV}$, as predicted by many model and QCD sum rule studies, should have been easily detectable in these analyses.

Among the channels where current results rule out the possibility of a bound, nonmolecular ground state is the $I=0, J^{P}=1^{+} u d \bar{c} \bar{b}$ channel. This conclusion stands in contrast to that reached in our earlier study of this channel [9], where indications for possible modest tetraquark

\footnotetext{
${ }^{1}$ For the single ensemble studied in this work, with heavierthan-physical pion mass $(192 \mathrm{MeV})$, we find the $J^{P}=1^{+} u d \bar{b} \bar{b}$ ground state to lie $\sim 113 \mathrm{MeV}$ below its lowest two-meson, $B B^{*}$, threshold and the $\ell s \bar{b} \bar{b}$ ground state to lie $\sim 36 \mathrm{MeV}$ below its lowest two-meson, $B_{s} B^{*}$, threshold. We emphasize that results at additional $m_{\pi}$ are required to extrapolate these binding energies to physical $m_{\pi}$, where increased binding is expected. This is the subject of an ongoing study, the results of which will be reported elsewhere.
}

binding were found, albeit based on rather short, late-time plateau signals, and with worries about possible FV effects for the ensemble with $m_{\pi}=164 \mathrm{MeV}$, where $m_{\pi} L=2.4$. The desirability of studying this channel at larger volumes, in order to more strongly test the bound state interpretation of the ground state results, was stressed already in Ref. [9], and the current study provides precisely such a largervolume extension, one which, moreover, has the advantage of the significantly improved box-sink analysis methodology. The indications of binding seen in the earlier study do not survive the improved, larger-volume, box-sink analysis: the ground-state mass is found to lie, clearly slightly above, rather than below, $D B^{*}$ threshold. The very good associated ground-state effective-mass plateau is shown in the right-hand panel of Fig. 9 in Appendix D. We conclude that there is, in fact, no nonmolecular bound tetraquark state in this channel, and that the late-time behavior of the earlier wall-local results was likely affected by the late-wall-local plateauing problem discussed in detail for the $B_{c}$ and doubly bottom $\overline{3}_{F}, J^{P}=1^{+}$channels in Sec. IV above.

Turning to a comparison to the results of other approaches, we note first that the predictions of the heavy-quark-symmetry approach [6,7] for the binding energies in the $I=0, J^{P}=1^{+} u d \bar{b} \bar{b}$ and $J^{P}=1^{+} \ell s \bar{b} \bar{b}$ channels, as well as for an absence of binding in the $J^{P}=0^{+}$and $1^{+}, I=0 u d \bar{c} \bar{b}$ and $J^{P}=0^{+}$and $1^{+} \ell s \bar{c} \bar{b}$ channels, are all compatible with current lattice results.

We turn next to tests of QCD sum rule and model predictions. As stressed by the authors of the various model tetraquark studies, with the parameters of the models already fully determined in earlier nonexotic sector studies, the predictions for tetraquark binding are parameter free and hence allow for highly nontrivial tests of the underlying models. In cases where multiple studies of the same model exist, we focus, where possible, on those in which numerical convergence of the prediction for the ground state energy has been demonstrated, taking these to supercede earlier, less complete studies, whether by the same or different authors.

The earliest of the chiral quark model (ChQM) studies, Ref. [21], employs a model with pseudoscalar nonet exchange potentials acting between light quarks only and color Coulomb and pairwise linear confinement potentials acting between all quarks. The resulting $I=0, J^{P}=1^{+} u d \bar{b} \bar{b}$ binding, $497 \mathrm{MeV}$, far exceeds that allowed by lattice results, thus ruling out this implementation of the ChQM approach. Apart from Ref. [39], all other ChQM studies we are aware of include octet (or nonet) pseudoscalar exchange potentials and scalar exchange potentials, both acting between light quarks only, pairwise or multibody confinement potentials, acting between all quarks, and, in addition to the color Coulomb part of the effective OGE interaction, the associated color-spindependent hyperfine interaction, again acting between all quarks. Specific implementations differ, especially with 
regard to the form of the effective scalar exchange(s). In Ref. [28] $u, d$ and $s$ quarks are assumed to interact via the exchange of a full nonet of scalar mesons. Other implementations include only the exchange of the $\sigma$, either in "SU(3)" form, where the exchange is restricted to $u, d$ and $s$ quarks $[23,25,29,31,35,36,38]$, or " $\mathrm{SU}(2)$ " form, where it is restricted further to $u$ and $d$ quarks only [30,35,41]. A final ChQM implementation, used to study the $I=0, J^{P}=0^{+}$ and $1^{+} u d \bar{s} \bar{b}$ channels [39], is the quark delocalization color screening model (QDCSM), which retains pseudoscalar exchange between light quarks and OGE between all quarks, but includes no scalar meson exchange, instead employing a modified confinement form that allows two-center cluster configurations with quark delocalization between the clusters. The scalar-nonet implementation is ruled out by its $32 \mathrm{MeV}$ prediction for the $I=0, J^{P}=1^{+} u d \bar{b} \bar{b}$ binding, which lies well below the range, $\sim 100-180 \mathrm{MeV}$, allowed by current lattice results. The $\mathrm{SU}(2)$ implementation is also ruled out, this time by significant overbindings relative to lattice results: 318 [41] or $404 \mathrm{MeV}$ [30] for the $I=0, J^{P}=$ $1^{+} u d \bar{b} \bar{b}$ channel and 178 and $199 \mathrm{MeV}$ [41] for the $I=0$, $J^{P}=0^{+}$and $1^{+} u d \bar{c} \bar{b}$ channels (which our results show to be at most weakly unbound). The $\mathrm{SU}(3)$ implementation is similarly ruled out, with predicted $I=0, J^{P}=1^{+} u d \bar{b} \bar{b}$ bindings of 341 [23], 214 [29], 217 [31], 278 [36] or 359 [38] $\mathrm{MeV}, I=0, J^{P}=0^{+}$and $1^{+} u d \bar{c} \bar{b}$ bindings of $136 \pm 12$ and $171 \pm 12 \mathrm{MeV}$ [36] or 178 and $217 \mathrm{MeV}$ [38], and $I=0, J^{P}=0^{+}$and $1^{+} u d \bar{s} \bar{b}$ bindings of 70 and $68 \mathrm{MeV}$ [35] (our lattice results again show the ground states to be at most weakly bound in these channels). Finally, the QCDSM implementation predicts bindings of 74 and $58 \mathrm{MeV}$ for the $I=0, J^{P}=0^{+}$and $1^{+} u d \bar{s} \bar{b}$ channels and hence is also ruled out by the absence of such deep binding in our lattice results. We conclude that all implementations of the ChQM approach are ruled out by current lattice results, and hence that the ChQM framework does not provide a reliable phenomenological representation of QCD in the low-energy regime.

The results of nonchiral models fare much better when tested against lattice results. Multiple nonrelativistic quark model studies of tetraquark channels exist using the AL1 [20] and Bhaduri (or BCN) [97] potentials. These potentials are characterized by pairwise linear confinement, color Coulomb and regularized OGE hyperfine interactions. The AL1 model is a variation of the BCN model in which the strict OGE relation between the strengths of the color Coulomb and hyperfine interactions has been relaxed. Reference [20] also investigates three additional models (the AL2, AP1 and AP2 models), characterized by alterations to the radial dependence of the AL1 confinement potential and/or the regularization of the AL1 OGE potentials. Predictions for binding in the $I=0$, $J^{P}=1^{+} u d \bar{b} \bar{b} ; J^{P}=1^{+} \ell s \bar{b} \bar{b} ; I=0, J^{P}=0^{+}$and $1^{+}$, $u d \bar{c} \bar{b}$; and $I=0, J^{P}=1^{+} u d \bar{c} \bar{c}$ channels exist for all these models, as well as for the Godfrey-Isgur-Capstick (GIC) model $[98,99]$, a "relativized" variant of this class of model. BCN results may be found in Refs. [17,19,20, 24,26,29,30], AL1 results in Refs. [20,24,34,40], AL2, AP1 and AP2 results in Ref. [20], and GIC results in Ref. [42]. Predictions for binding in the $I=0$, $J^{P}=1^{+} u d \bar{b} \bar{b} ; J^{P}=1^{+} \ell s \bar{b} \bar{b} ; I=0, J^{P}=0^{+} u d \bar{c} \bar{b}$; and $I=0, J^{P}=1^{+} u d \bar{c} \bar{b}$ channels, obtained from the nonrelativistic BCN, AL1, AL2, AP1 and AP2 models, range from 131 to $167 \mathrm{MeV}[19,20,24,26,29], 40$ to $61 \mathrm{MeV}$ [20], 0 to $23 \mathrm{MeV}[20,34]$ and 0 to $23 \mathrm{MeV}$ [20,34], respectively. Taking the $\sim 20-35 \mathrm{MeV}$ variations with model choice for these channels seen in Ref. [20] as a measure of the uncertainties associated with the specifics of the implementations of the interactions in this class of model, one sees that the predictions are compatible within errors with current lattice results. Future lattice studies, using larger-volume ensembles to better quantify possible residual $\mathrm{FV}$ effects, will be useful for sharpening these tests for the $J^{P}=0^{+}$and $1^{+}, I=0 u d \bar{c} \bar{b}$ channels, where the results of this paper show no evidence for binding while the most recent AL1 model study [34] finds modest $23 \mathrm{MeV}$ bindings for both. The relativized GIC model, in contrast, predicts only $54 \mathrm{MeV}$ binding in the $I=0, J^{P}=1^{+} u d \bar{b} \bar{b}$ channel and no binding in the $J^{P}=1^{+} \ell s \bar{b} \bar{b}$ channel [42] and hence is ruled out by lattice results.

Another model approach ruled out by lattice results, specifically those for the $I=0 u d \bar{c} \bar{b}$ channels, is that of the LAMP model. In this approach, an ansatz for a relativistic confinement potential is employed in the multiquark sector. Taking the $B D$ interaction studied in Ref. [32] to be specific, linearly rising mean-field confinement potentials for the $B$ and $D$ are merged into a one-body, two-well, W-shaped confinement potential for the $B D$ system by truncating the individual $B$ and $D$ wells at the midpoint between their centers. Such a potential allows for the delocalization of an initially single-well light-quark wave function into the second of the two potential wells. The optimal well-center separation and degree of delocalization are determined by a variational minimization. The result, in which the effect of the OGE hyperfine interaction has been neglected, is a $B D$ binding of $155 \mathrm{MeV}$, clearly incompatible with the lattice results above. This rules out the LAMP ansatz for the effective confining interaction.

Turning to tests of QCD sum rule results, we see that recent predictions for very deep tetraquark binding (by $569 \pm 260 \mathrm{MeV}[48]$ in the $I=0, J^{P}=1^{+} u d \bar{b} \bar{b}$ channel, $477 \pm 250 \mathrm{MeV}$ [53] in the $J^{P}=1^{+} \ell s \bar{b} \bar{b}$ channel, $485 \pm$ $150 \mathrm{MeV}[48]$ in the $I=0, J^{P}=0^{+} u d \bar{c} \bar{b}$ channel, $407 \pm$ $160 \mathrm{MeV}$ [49], $467 \pm 150 \mathrm{MeV}$ [53] and $200 \pm 130 \mathrm{MeV}$ [54] in the $J^{P}=0^{+} \ell s \bar{c} \bar{b}$ channel, $180 \pm 110 \mathrm{MeV}$ [54] in the $J^{P}=1^{+} \ell s \bar{c} \bar{b}$ channel, and $394 \pm 170 \mathrm{MeV}$ [52] in the $I=0, J^{P}=0^{+} u d \bar{s} \bar{b}$ channel) are not compatible, within quoted uncertainties, with current lattice results. Other sum rule predictions $(400 \pm 300 \mathrm{MeV}[43,44]$, 
$80 \pm 80 \mathrm{MeV}$ [46] and $240 \pm 150 \mathrm{MeV}$ [55] in the $I=0, \quad J^{P}=1^{+} u d \bar{b} \bar{b}$ channel, $290 \pm 300 \mathrm{MeV}$ [44], $140 \pm 80 \mathrm{MeV}$ [46] and $180 \pm 160 \mathrm{MeV}$ [55] in the $J^{P}=1^{+} \ell s \bar{b} \bar{b}$ channel, $5 \pm 100$ and $60 \pm 100 \mathrm{MeV}$ [45] in the $I=0, J^{P}=0^{+}$and $J^{P}=1^{+} u d \bar{c} \bar{b}$ channels) are compatible with lattice results within errors, though, with the exception of the $I=0, J^{P}=1^{+} u d \bar{b} \bar{b}$ prediction of Ref. [46], with significantly higher central values. It is not clear whether the above disagreements with lattice results are due to shortcomings associated with the use of the SVZ ansatz, inaccuracies in the factorization approximation for some of the higher dimension condensates, a need for updates to OPE input (e.g., the quark condensate, where precise results from FLAG are now available [100]), or other aspects of the sum rule implementations. On this last point it is worth noting the discrepancy between the predictions, $80 \pm 80 \mathrm{MeV}$ [46] and $569 \pm 260 \mathrm{MeV}$ [48], for binding in the $I=0, J^{P}=1^{+} u d \bar{b} \bar{b}$ channel obtained from analyses employing the same interpolating operator, essentially identical OPE input, and both truncating the OPE at the same dimension, $D=10$. The predictions, $142 \pm 80 \mathrm{MeV}$ [46] and $477 \pm 250 \mathrm{MeV}$ [53], for binding in the $J^{P}=1^{+} \ell s \bar{b} \bar{b}$ channel also come from analyses using the same interpolating operator, essentially identical OPE input and truncation of the OPE at the same dimension, $D=10$. A similar discrepancy exists in the $I=0, J^{P}=0^{+} u d \bar{c} \bar{b}$ channel where very different ground state masses, $7140 \pm 100 \mathrm{MeV}[45]$ and $6660 \pm 150 \mathrm{MeV}$ [48], are predicted by analyses using the same interpolating operator and similar OPE input, differing only in the dimension at which the OPE was truncated $(D=8$ in Ref. [45] and $D=10$ in Ref. [48]). These observation make clear that significant, yet-to-be-fully quantified systematic uncertainties exist for applications of the conventional implementation of the QCD sum rule framework to the multiquark sector. The targets provided by current (and future) lattice results for tetraquark binding (or lack thereof) in the channels investigated in this paper should help in further investigations of these issues and have the potential to aid in the development of improved sum rule implementation strategies.

We close with a reminder of improvements/extensions to the present study either already in progress or planned for the near future. The first is the completion of the extension of the $m_{\pi} \simeq 192 \mathrm{MeV}$ box-sink analysis reported here to additional $48^{3} \times 64$ ensembles having both lighter and heavier $m_{\pi}$. This will allow us to update and sharpen our previous physical-point extrapolations for the bindings of the doubly bottom, $J^{P}=1^{+}, \overline{3}_{F}$ states, and further test nonchiral model predictions. This work is ongoing. The next step after that is to generate a new set of ensembles with even larger volume covering a similar range of $m_{\pi}$. This will allow us to carry out quantitative FV studies and extend the current analysis to channels, such as the doubly charmed channels, where shallow binding remains a possibility and experimental detection would be less challenging than in the doubly bottom sector.

\section{ACKNOWLEDGMENTS}

B. C., R. L., and K. M. are supported by grants from the Natural Sciences and Engineering Research Council of Canada, R. J.H. by the European Research Council (ERC) under the European Unions Horizon 2020 research and innovation programme through Grant Agreement No. 771971-SIMDAMA. All computations were carried out using a crucial allocation from Compute Canada on the Niagara supercomputer at Scinet.

\section{APPENDIX A: TUNING NRQCD}

We use the tree-level $\left(c_{i}=1\right)$, tadpole-improved, NRQCD action of [96], defined by the symmetric time evolution [101],

$$
G(x, t+1)=\left(1-\frac{\delta H}{2}\right)\left(1-\frac{H_{0}}{2 n}\right)^{n} U_{t}\left(x, t_{0}\right)^{\dagger}\left(1-\frac{H_{0}}{2 n}\right)^{n}\left(1-\frac{\delta H}{2}\right) G(t),
$$

where

$$
\begin{aligned}
H_{0} & =-c_{0} \frac{1}{2 M_{0}} \Delta^{2} \\
H_{I} & =\left(-c_{1} \frac{1}{8 M_{0}^{2}}-c_{6} \frac{1}{16 n M_{0}^{2}}\right)\left(\Delta^{2}\right)^{2}+c_{2} \frac{i}{8 M_{0}^{2}}(\tilde{\Delta} \cdot \tilde{E}-\tilde{E} \cdot \tilde{\Delta})+c_{5} \frac{\Delta^{4}}{24 M_{0}} \\
H_{D} & =-c_{3} \frac{1}{8 M_{0}^{2}} \sigma \cdot(\tilde{\Delta} \times \tilde{E}-\tilde{E} \times \tilde{\Delta})-c_{4} \frac{1}{8 M_{0}} \sigma \cdot \tilde{B} \\
\delta H & =H_{I}+H_{D} .
\end{aligned}
$$

A tilde indicates that an improved version has been used (see e.g., [102] for the tadpole-improved derivative terms), and $M_{0}$ is a bare parameter that we tune to recover the spinaveraged kinetic mass of the connected $\eta_{b}$ and $\Upsilon$. We use the fourth root of the plaquette for the tadpole improvement and a value of $n=4$ for the stability parameter.

For the tuning we use Coulomb gauge-fixed wallsources, and we implement the momenta by solving one 
TABLE V. Twist angles, chosen so the $(a p)^{2}$ values are evenly spaced between $(0,0,0)$ and $(1,1,1)$.

\begin{tabular}{llcc}
\hline \hline$(\theta, \theta, \theta)$ & $(a p)^{2}$ & $(\theta, \theta, \theta)$ & $(a p)^{2}$ \\
\hline$(0,0,0)$ & 0 & $\left(\frac{\sqrt{5}}{3}, \frac{\sqrt{5}}{3}, \frac{\sqrt{5}}{3}\right)$ & 0.064255 \\
$\left(\frac{1}{3}, \frac{1}{3}, \frac{1}{3}\right)$ & 0.012851 & $\left(\frac{\sqrt{6}}{3}, \frac{\sqrt{6}}{3}, \frac{\sqrt{6}}{3}\right)$ & 0.077106 \\
$\left(\frac{\sqrt{2}}{3}, \frac{\sqrt{2}}{3}, \frac{\sqrt{2}}{3}\right)$ & 0.025702 & $\left(\frac{\sqrt{7}}{3}, \frac{\sqrt{7}}{3}, \frac{\sqrt{7}}{3}\right)$ & 0.089957 \\
$\left(\frac{\sqrt{3}}{3}, \frac{\sqrt{3}}{3}, \frac{\sqrt{3}}{3}\right)$ & 0.038553 & $\left(\frac{\sqrt{8}}{3}, \frac{\sqrt{8}}{3}, \frac{\sqrt{8}}{3}\right)$ & 0.102808 \\
$\left(\frac{2}{3}, \frac{2}{3}, \frac{2}{3}\right)$ & 0.051404 & $(1,1,1)$ & 0.115659 \\
\hline \hline
\end{tabular}

of the $\mathrm{b}$ propagators on a partially twisted boundary $[80,81]$. This is practically implemented by applying a $U(1)$ phase to the gauge field [103],

$$
U_{\mu}(x) \rightarrow e^{i 2 \pi \theta_{\mu} / L_{\mu}} U_{\mu}(x)
$$

which yields $p_{\mu}=\frac{2 \pi}{L_{\mu}} \theta_{\mu}$. The benefit of this approach is that one can stay within the same irrep for arbitrary momenta, smoothly interpolating discretization and finite-volume effects. We choose to twist equally in all directions to minimize the rotation-breaking hypercubic artifacts and the effects introduced by the twisting. The momenta we use is listed in Table V, and the effective masses for these twists is shown in Fig. 7.

Once we have computed the correlation functions along all our directions we simultaneously fit them to the Ansatz,

$$
\begin{aligned}
& A\left(1+(a p)^{2} B+C\left((a p)^{2}\right)^{2}\right) \\
& \quad \times \exp \left[-t\left(\left(a M_{1}\right)+\frac{1}{2 a M_{K}}(a p)^{2}\right)\right],
\end{aligned}
$$

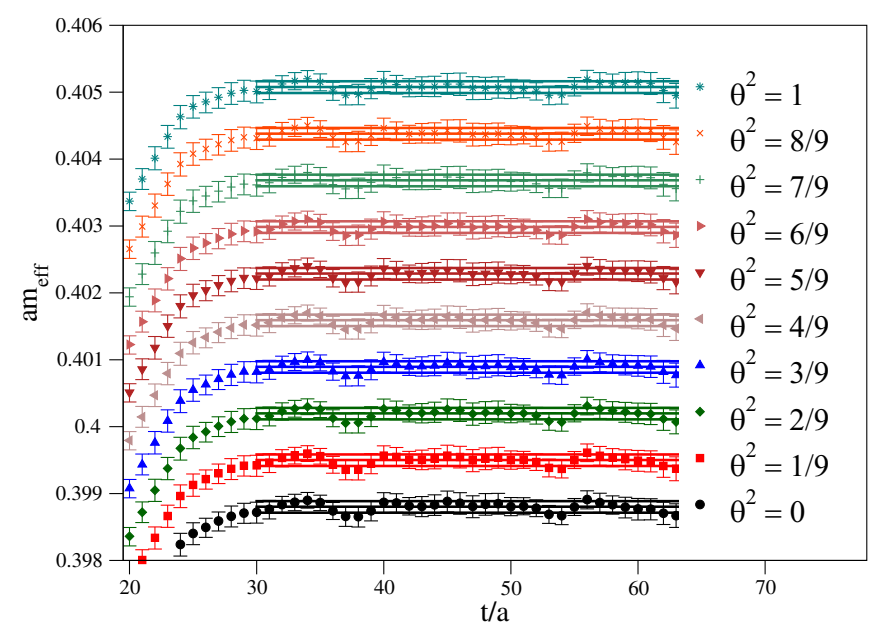

(a) Effective masses

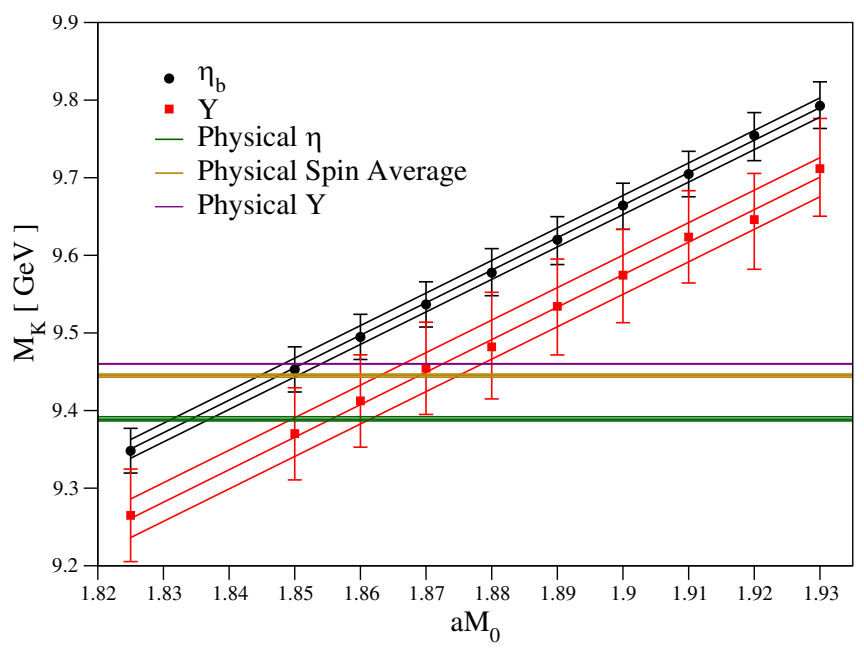

FIG. 8. Kinetic mass dependence on the bare mass $a M_{0}$. The fit is correlated and constrained to have the same slope for the $\eta_{b}, \Upsilon$ and spin average.

where $a M_{1}$ is the mass of the ground state and $a M_{K}$ is the kinetic mass. This enables us to describe our data simply by five fit parameters.

We note that, as in Ref. [104], the kinetic mass ordering is inverted compared to nature. This situation can be improved by the inclusion of yet-higher-order $\left(1 / M^{3}\right)$ terms in the NRQCD Hamiltonian [105], specifically the parameter $c_{7}$. However, we follow the approach of [104] and tune to the spin average of these kinetic masses instead. Using HPQCD's estimate [104] for the spin-averaged mass, $9.445(2) \mathrm{GeV}$, and the value $a^{-1}=2.194 \mathrm{GeV}$ [86] for our lattice spacing, we find the physical $\mathrm{b}$ quark corresponds to a bare mass $a M_{0}=1.864$. This tuning is obtained by measuring the kinetic masses for several bare masses and fitting their dependence linearly, as is shown in Fig. 8.

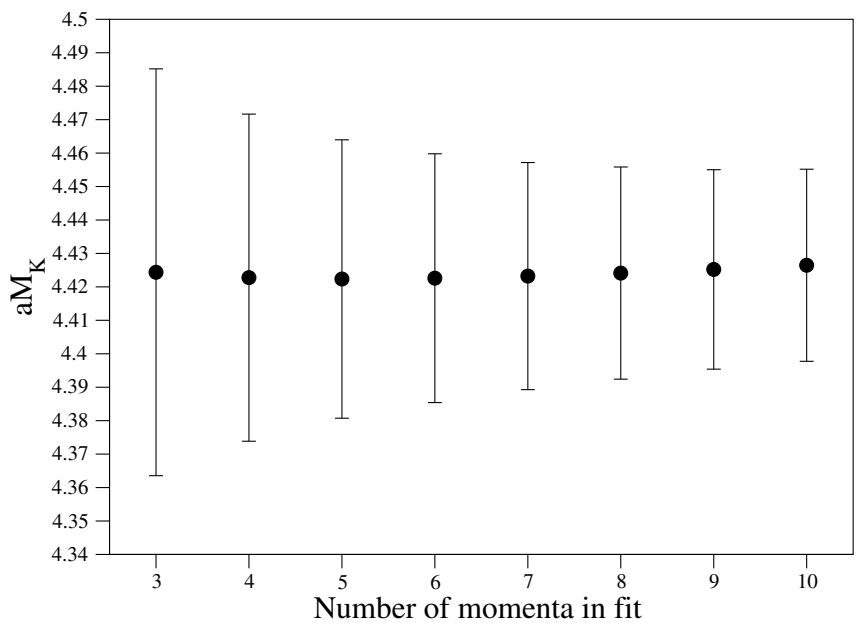

(b)

FIG. 7. Left: Effective masses for each twist angle and corresponding fit for $a M_{0}=1.88$. Right: Stability and error reduction as the number of momenta is increased. 
When we compute our heavy-light mesons we pack the backward and forward propagating NRQCD propagators into one as the NRQCD propagator has only two Dirac indices whereas the full light, strange, and charm propagators have four,

$$
S(x)=\left(\begin{array}{cc}
S^{\mathrm{fwd}}(x) & 0 \\
0 & S^{\mathrm{bwd}}(x)
\end{array}\right) .
$$

We also apply antiperiodic boundary conditions in time to the NRQCD propagators.

\section{APPENDIX B: MESON MASSES}

As we use Coulomb gauge-fixed wall sources our amplitudes are partially related to physical matrix elements but are polluted by the wall source [77]. If we consider for example the pseudoscalar $J^{P}=0^{-}$meson channel, we can use any of the following eight correlation functions at large asymptotic times:

$$
\begin{aligned}
& C_{P P}^{W L}(t)=\frac{P^{W} P^{L}}{2 m_{P}}\left(e^{-m_{P} t}+e^{-m_{P}\left(L_{t}-t\right)}\right), \\
& C_{A A}^{W L}(t)=\frac{A^{W} A^{L}}{2 m_{P}}\left(e^{-m_{P} t}+e^{-m_{P}\left(L_{t}-t\right)}\right), \\
& C_{A P}^{W L}(t)=\frac{A^{W} P^{L}}{2 m_{P}}\left(e^{-m_{P} t}-e^{-m_{P}\left(L_{t}-t\right)}\right), \\
& C_{P A}^{W L}(t)=\frac{P^{W} A^{L}}{2 m_{P}}\left(e^{-m_{P} t}-e^{-m_{P}\left(L_{t}-t\right)}\right), \\
& C_{P P}^{W W}(t)=\frac{P^{W} P^{W}}{2 m_{P}}\left(e^{-m_{P} t}+e^{-m_{P}\left(L_{t}-t\right)}\right), \\
& C_{A A}^{W W}(t)=\frac{A^{W} A^{W}}{2 m_{P}}\left(e^{-m_{P} t}+e^{-m_{P}\left(L_{t}-t\right)}\right), \\
& C_{A P}^{W W}(t)=\frac{A^{W} P^{W}}{2 m_{P}}\left(e^{-m_{P} t}-e^{-m_{P}\left(L_{t}-t\right)}\right), \\
& C_{P A}^{W W}(t)=\frac{P^{W} A^{W}}{2 m_{P}}\left(e^{-m_{P} t}-e^{-m_{P}\left(L_{t}-t\right)}\right) .
\end{aligned}
$$

Here we use $P$ to denote the operator with a $\gamma_{5}$-insertion and $A$ to denote the temporal axial current operator. Using these sources means, for example, that $C_{P A}^{W L}$ is not equal to $C_{A P}^{W L}$. One can, however, clearly fit all eight correlators simultaneously with five fit parameters. One can similarly analyze correlators involving the $V_{i}=\gamma_{i}$ and $T_{i t}=\gamma_{i} \gamma_{t}$ currents for $J^{P}=1^{-}$states or $I$ and $\gamma_{t}$ for $J^{P}=0^{+}$states. The same fit form can be used when the sink is nonlocal too, although neither matrix element will correspond to a physical one. We use these fits to determine the masses of mesons not containing a $\mathrm{b}$ quark. This fit is not possible for mesons containing b quarks as $C_{A A}=C_{P P}$ at our level of heavy-light approximation.

\section{APPENDIX C: TABLES OF RESULTS}

In this work we use simple, local operators of the form,

$$
M=(\bar{\psi} \Gamma \phi)
$$

to determine the masses of ordinary meson states. The results, in lattice units, and with the additive offset in the case of mesons containing a $\mathrm{b}$ quark, are given in Table VI. Where there are several $\Gamma$ insertions listed, a simultaneous fit of the form of Eq. (B1) was used; otherwise a correlated single-cosh fit was performed on the one channel. As some of the particles listed in the table are resonances, the ground-state masses obtained from the associated localoperator correlators should not be directly compared to the masses of these resonances in nature. To simplify notation,

TABLE VI. Table of results for the ground-state effective masses associated with the simple, local meson operators used in this work.

\begin{tabular}{lclccl}
\hline \hline State & $\Gamma$ & \multicolumn{1}{c}{$a m$} & State & $\Gamma$ & $a(m+\Delta)$ \\
\hline$\pi$ & $\gamma_{5}, \gamma_{t} \gamma_{5}$ & $0.0880(8)$ & $B$ & $\gamma_{5}$ & $0.4546(6)$ \\
$K$ & $\gamma_{5}, \gamma_{t} \gamma_{5}$ & $0.2313(2)$ & $B^{*}$ & $\gamma_{i}$ & $0.4712(6)$ \\
$K_{0}^{*}(700)$ & $I, \gamma_{t}$ & $0.402(3)$ & $B_{I}$ & $I$ & $0.590(5)$ \\
$K^{*}(892)$ & $\gamma_{i}, \gamma_{i} \gamma_{t}$ & $0.401(9)$ & $B_{s}$ & $\gamma_{5}$ & $0.4926(3)$ \\
$D$ & $\gamma_{5}, \gamma_{t} \gamma_{5}$ & $0.8585(6)$ & $B_{s}^{*}$ & $\gamma_{i}$ & $0.5116(3)$ \\
$D^{*}$ & $\gamma_{i}, \gamma_{i} \gamma_{t}$ & $0.9155(6)$ & $B_{c}$ & $\gamma_{5}$ & $0.92011(8)$ \\
$D_{0}^{*}(2300)$ & $I, \gamma_{t}$ & $1.016(5)$ & $B_{c}^{*}$ & $\gamma_{i}$ & $0.9429(1)$ \\
\hline \hline
\end{tabular}

TABLE VII. Eigenvalues and fit parameters for the $I=0$, $J^{P}=0^{+}$and $1^{+} u d \bar{c} \bar{b}$ channels.

\begin{tabular}{clcc}
\hline \hline$J^{P}$ & $a(m+\Delta)$ & fit (low,high) & $\chi^{2} /$ d.o.f. \\
\hline $0^{+}$ & $1.3208(10)$ & $(7,18)$ & 1.2 \\
& $1.3878(11)$ & $(5,18)$ & 1.1 \\
& $1.606(20)$ & $(6,12)$ & 0.9 \\
$1^{+}$ & $1.3356(10)$ & $(7,17)$ & 1.2 \\
& $1.3731(13)$ & $(7,17)$ & 0.8 \\
& $1.3864(12)$ & $(6,18)$ & 0.9 \\
& $1.632(12)$ & $(5,9)$ & 1.1 \\
& $1.680(15)$ & $(5,10)$ & 0.6 \\
\hline \hline
\end{tabular}

TABLE VIII. Eigenvalues and fit parameters for the $I=0$, $J^{P}=1^{+} s c \bar{b} \bar{b}$ channel.

\begin{tabular}{cccc}
\hline \hline$J^{P}$ & $a(m+\Delta)$ & fit (low,high) & $\chi^{2} /$ d.o.f. \\
\hline $1^{+}$ & $1.4323(4)$ & $(8,22)$ & 1.2 \\
& $1.4422(5)$ & $(13,22)$ & 0.8 \\
& $1.4448(6)$ & $(16,25)$ & 0.6 \\
& $1.4585(5)$ & $(13,22)$ & 0.8 \\
& $1.5263(184)$ & $(14,22)$ & 0.6 \\
\hline \hline
\end{tabular}


TABLE IX. Eigenvalues and fit parameters for the $J^{P}=0^{+}$and $1^{+} \ell s \bar{c} \bar{b}$ channels.

\begin{tabular}{cccc}
\hline \hline$J^{P}$ & $a(m+\Delta)$ & fit (low,high) & $\chi^{2} /$ d.o.f. \\
\hline $0^{+}$ & $1.3471(8)$ & $(8,27)$ & 1.2 \\
& $1.3573(9)$ & $(10,19)$ & 1.0 \\
& $1.417(1)$ & $(8,18)$ & 0.8 \\
& $1.445(2)$ & $(10,15)$ & 1.5 \\
$1^{+}$ & $1.3656(8)$ & $(7,20)$ & 1.2 \\
& $1.3746(8)$ & $(8,18)$ & 1.1 \\
& $1.406(1)$ & $(8,18)$ & 1.2 \\
& $1.414(1)$ & $(7,20)$ & 1.2 \\
& $1.423(1)$ & $(8,18)$ & 1.0 \\
& $1.59(1)$ & $(8,18)$ & 1.0 \\
\hline \hline
\end{tabular}

TABLE X. Eigenvalues and fit parameters for the $I=0, J^{P}=$ $0^{+}$and $1^{+} u d \bar{s} \bar{b}$ channels.

\begin{tabular}{clcc}
\hline \hline$J^{P}$ & $a(m+\Delta)$ & fit (low,high) & $\chi^{2} /$ d.o.f. \\
\hline $0^{+}$ & $0.6939(7)$ & $(7,25)$ & 1.1 \\
& $0.8802(19)$ & $(7,16)$ & 1.1 \\
& $1.023(17)$ & $(6,11)$ & 0.6 \\
$1^{+}$ & $0.7100(9)$ & $(7,20)$ & 1.0 \\
& $0.8639(19)$ & $(7,16)$ & 1.0 \\
& $0.8695(52)$ & $(10,16)$ & 1.0 \\
& $1.038(17)$ & $(6,12)$ & 0.5 \\
& $1.163(12)$ & $(5,12)$ & 1 \\
\hline \hline
\end{tabular}

TABLE XI. Eigenvalues and fit parameters for the $I=0$, $J^{P}=0^{+}$and $1^{+} u d \bar{s} \bar{c}$ channels.

\begin{tabular}{cccc}
\hline \hline$J^{P}$ & $a(m+\Delta)$ & fit (low,high) & $\chi^{2} /$ d.o.f. \\
\hline $0^{+}$ & $1.0949(6)$ & $(7,17)$ & 1.3 \\
& $1.321(2)$ & $(4,13)$ & 0.7 \\
& $1.423(2)$ & $(3,9)$ & 0.8 \\
$1^{+}$ & $1.1536(7)$ & $(8,19)$ & 1.0 \\
& $1.266(2)$ & $(8,22)$ & 1.0 \\
& $1.321(2)$ & $(6,18)$ & 1.0 \\
& $1.465(2)$ & $(6,14)$ & 0.7 \\
& $1.590(1)$ & $(4,13)$ & 0.5 \\
\hline \hline
\end{tabular}

we have, nonetheless, used the name of the lowest-lying resonance in the channel to label the channel itself in these cases, trusting this will not lead to any confusion.
TABLE XII. Eigenvalues and fit parameters for the $I=0$, $J^{P}=1^{+} u c \bar{b} \bar{b}$ channel.

\begin{tabular}{clcc}
\hline \hline$J^{P}$ & $a(m+\Delta)$ & fit (low,high) & $\chi^{2} /$ d.o.f. \\
\hline $1^{+}$ & $1.3926(6)$ & $(6,25)$ & 0.7 \\
& $1.4015(11)$ & $(10,25)$ & 1.2 \\
& $1.4094(13)$ & $(10,17)$ & 1.3 \\
& $1.4196(13)$ & $(10,17)$ & 1.3 \\
\hline \hline
\end{tabular}

Throughout this work we use the shorthand name $B_{I}$ for the scalar, $J^{P}=0^{+}$B meson.

Tables VII through VIII present the results for the lowest resolvable GEVP eigenvalues in the ten tetraquark channels specified in the main text, obtained from correlated, single-exponential fits to the plateau regions of the corresponding optimised correlators. Entries in column 1 specify the quantum numbers of the state. Those in columns 2, 3 and 4 give the corresponding eigenvalue (in lattice units), the fit window interval (in lattice units) and the associated $\chi^{2} /$ d.o.f. Fits for higher-lying excited states having reasonable $\chi^{2} /$ d.o.f. have been included even in cases where the fit window is relatively short since such results help identify those parts of the higher-lying twomeson spectrum having the largest couplings to the operators used in constructing the optimised correlators.

\section{APPENDIX D: EFFECTIVE MASS PLOTS}

If we consider our correlators $C(t)$ to behave like single exponentials at large $t$ (a reasonable expectation for heavy states) we can define an effective mass via

$$
a m_{\mathrm{eff}}(t)=\tanh ^{-1}\left(\frac{C(t-1)-C(t+1)}{C(t-1)+C(t+1)}\right) .
$$

This has the desirable property that $\tanh ^{-1}(x)$ is defined for all $x$ in $[-1,1]$ and provides a definition that is, empirically, better behaved for very noisy data than the more common log or $\cosh ^{-1}$ definitions, $\log \left(\frac{C(t)}{C(t+1)}\right)$ or $\cosh ^{-1}\left(\frac{C(t+1)+C(t-1)}{2 C(t)}\right)$, where fluctuations can lead to negative values of the ratios appearing in the arguments of the $\log 0 \cosh ^{-1}$, causing these definitions to break down.

Figures 9-12 show the resulting $J^{P}=0^{+}$and $1^{+}$ effective masses for the $u d \bar{c} \bar{b}, \ell s \bar{c} \bar{b}, u d \bar{s} \bar{b}$ and $u d \bar{s} \bar{c}$ channels, respectively. Figure 13, similarly, shows the effective masses for the $J^{P}=1^{+} u c \bar{b} \bar{b}$ and $s c \bar{b} \bar{b}$ channels. 

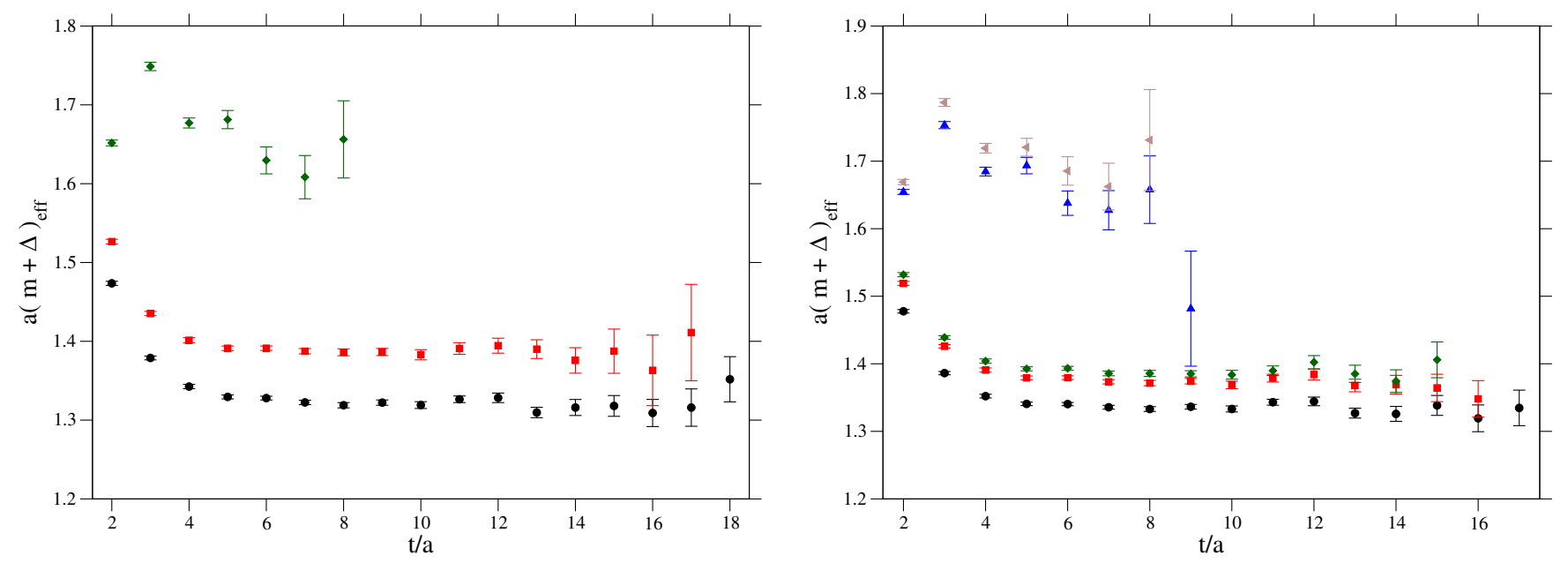

FIG. 9. Effective mass plots for the $I=0, J^{P}=0^{+}$(left) and $1^{+}$(right) $u d \bar{c} \bar{b}$ channels.
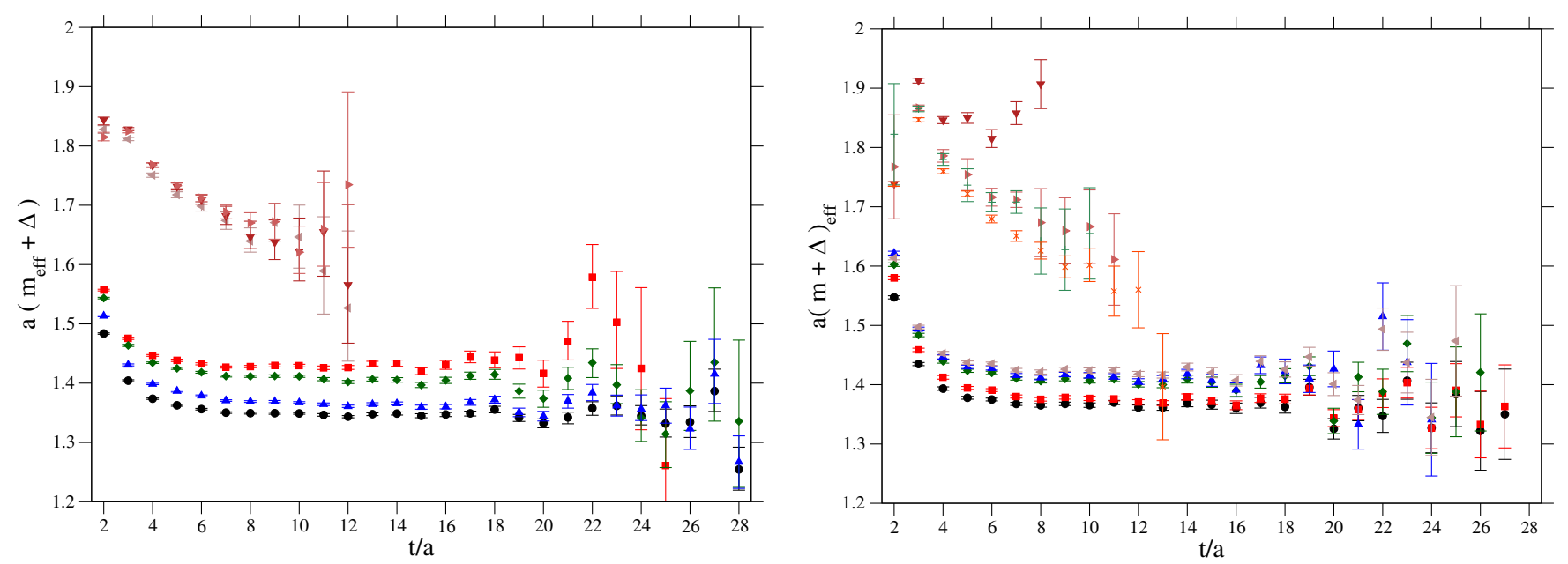

FIG. 10. Effective mass plots for the $J^{P}=0^{+}$(left) and $1^{+}$(right) $\ell s \bar{c} \bar{b}$ channels.
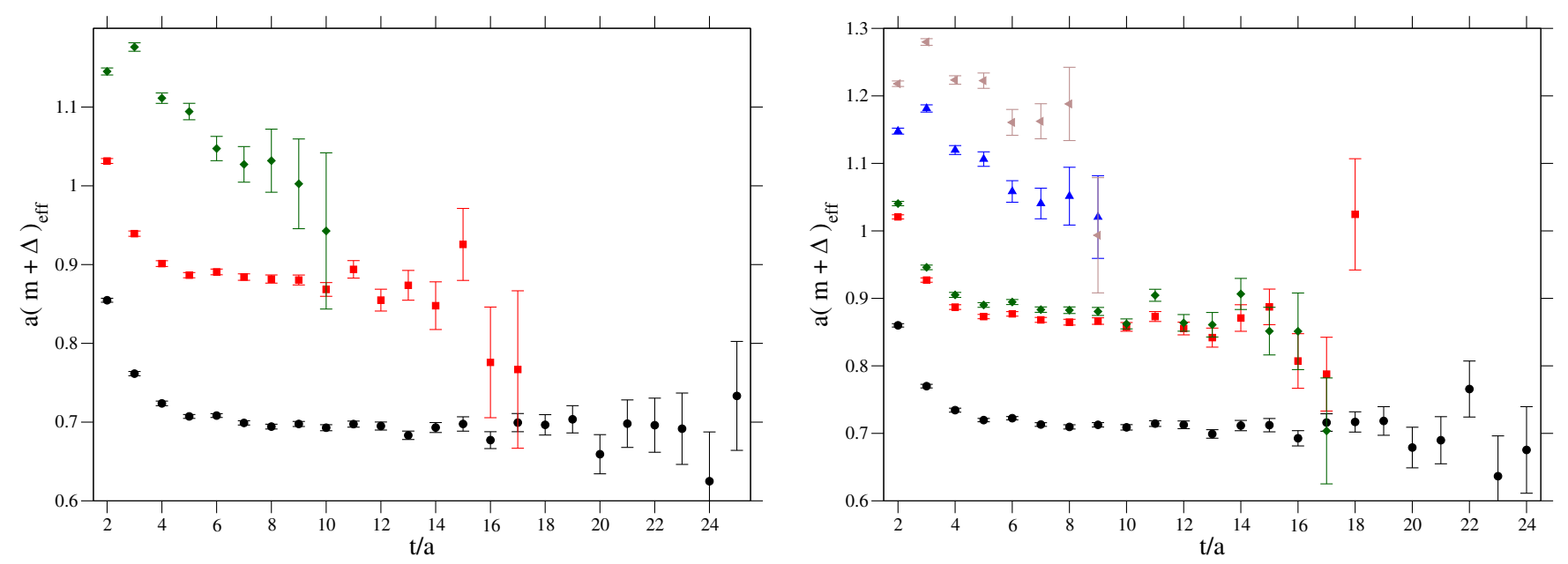

FIG. 11. Effective mass plots for the $I=0, J^{P}=0^{+}$(left) and $1^{+}$(right) $u d \bar{s} \bar{b}$ channels. 

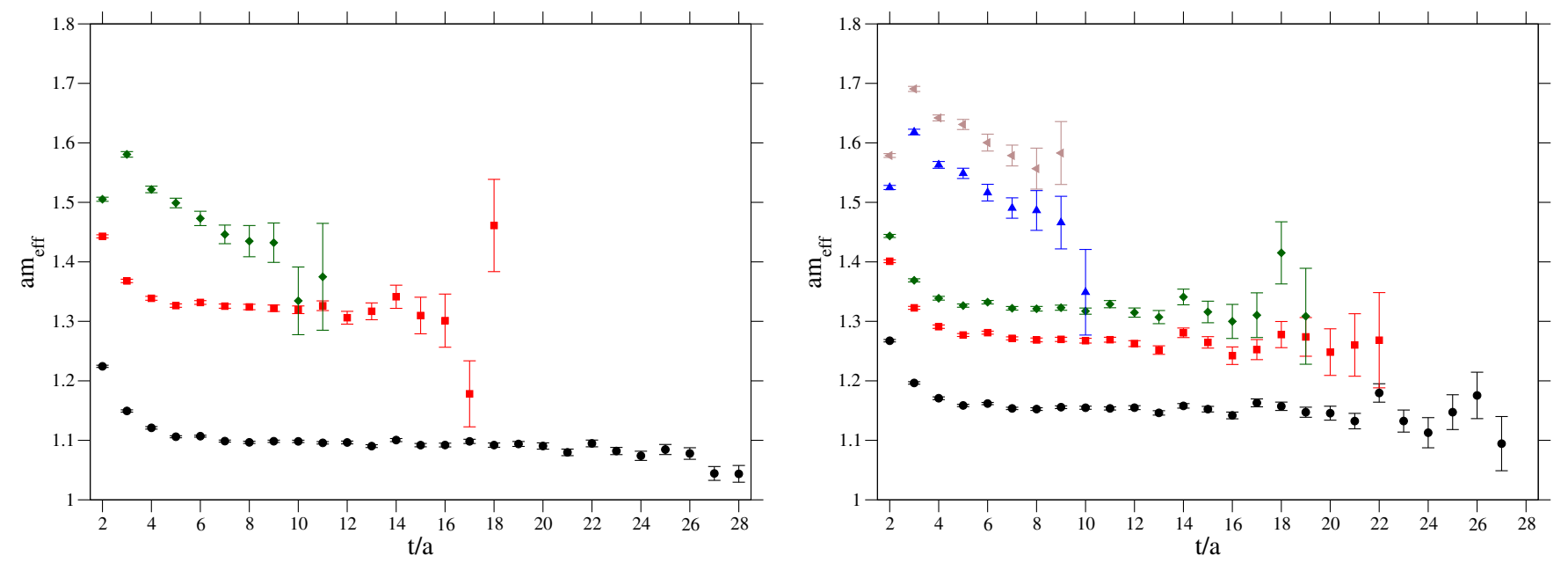

FIG. 12. Effective mass plots for the $I=0, J^{P}=0^{+}$(left) and $1^{+}$(right) $u d \bar{s} \bar{c}$ channels.
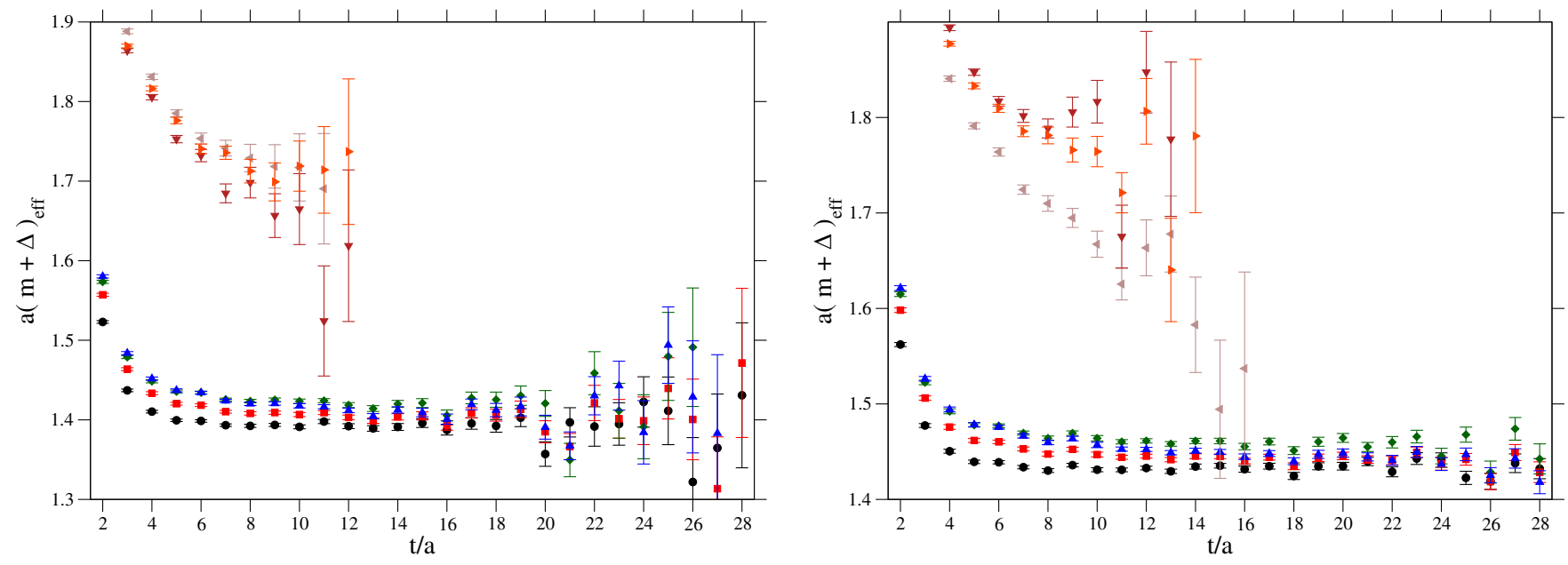

FIG. 13. Effective mass plots for the $J^{P}=1^{+} u c \bar{b} \bar{b}$ (left) and $s c \bar{b} \bar{b}$ (right) channels.

[1] P. Bicudo, K. Cichy, A. Peters, and M. Wagner, Phys. Rev. D 93, 034501 (2016).

[2] P. Bicudo, J. Scheunert, and M. Wagner, Phys. Rev. D 95, 034502 (2017).

[3] A. Francis, R. J. Hudspith, R. Lewis, and K. Maltman, Phys. Rev. Lett. 118, 142001 (2017).

[4] P. Junnarkar, N. Mathur, and M. Padmanath, Phys. Rev. D 99, 034507 (2019).

[5] L. Leskovec, S. Meinel, M. Pflaumer, and M. Wagner, Phys. Rev. D 100, 014503 (2019).

[6] E. J. Eichten and C. Quigg, Phys. Rev. Lett. 119, 202002 (2017).
[7] E. Braaten, L.-P. He, and A. Mohapatra, arXiv:2006 .08650 .

[8] Other arguments favoring the possibility of bound doubly bottom tetraquarks, may be found in Refs. [106,107].

[9] A. Francis, R. J. Hudspith, R. Lewis, and K. Maltman, Phys. Rev. D 99, 054505 (2019).

[10] R. Aaij et al. (LHCb Collaboration), Phys. Rev. Lett. 119, 112001 (2017).

[11] R. Aaij et al. (LHCb Collaboration), Phys. Rev. Lett. 121, 162002 (2018).

[12] V. Khachatryan et al. (CMS Collaboration), J. High Energy Phys. 05 (2017) 013. 
[13] T. Gershon and A. Poluektov, J. High Energy Phys. 01 (2019) 019.

[14] J. Ader, J. Richard, and P. Taxil, Phys. Rev. D 25, 2370 (1982).

[15] L. Heller and J. Tjon, Phys. Rev. D 35, 969 (1987).

[16] J. Carlson, L. Heller, and J. Tjon, Phys. Rev. D 37, 744 (1988).

[17] S. Zouzou, B. Silvestre-Brac, C. Gignoux, and J. Richard, Z. Phys. C 30, 457 (1986).

[18] H. J. Lipkin, Phys. Lett. B 172, 242 (1986).

[19] B. Silvestre-Brac and C. Semay, Z. Phys. C 57, 273 (1993).

[20] C. Semay and B. Silvestre-Brac, Z. Phys. C 61, 271 (1994).

[21] S. Pepin, F. Stancu, M. Genovese, and J. Richard, Phys. Lett. B 393, 119 (1997).

[22] D. Brink and F. Stancu, Phys. Rev. D 57, 6778 (1998).

[23] J. Vijande, F. Fernandez, A. Valcarce, and B. SilvestreBrac, Eur. Phys. J. A 19, 383 (2004).

[24] D. Janc and M. Rosina, Few Body Syst. 35, 175 (2004).

[25] J. Vijande, A. Valcarce, and K. Tsushima, Phys. Rev. D 74, 054018 (2006).

[26] J. Vijande, E. Weissman, A. Valcarce, and N. Barnea, Phys. Rev. D 76, 094027 (2007).

[27] D. Ebert, R. Faustov, V. Galkin, and W. Lucha, Phys. Rev. D 76, 114015 (2007).

[28] M. Zhang, H. Zhang, and Z. Zhang, Commun. Theor. Phys. 50, 437 (2008).

[29] J. Vijande, A. Valcarce, and N. Barnea, Phys. Rev. D 79, 074010 (2009).

[30] Y. Yang, C. Deng, J. Ping, and T. Goldman, Phys. Rev. D 80, 114023 (2009).

[31] T. Carames, A. Valcarce, and J. Vijande, Phys. Lett. B 699, 291 (2011).

[32] R. R. Silbar and T. Goldman, Int. J. Mod. Phys. E 23, 1450091 (2014).

[33] M. Karliner and J. L. Rosner, Phys. Rev. Lett. 119, 202001 (2017).

[34] T. F. Carams, J. Vijande, and A. Valcarce, Phys. Rev. D 99, 014006 (2019).

[35] X. Chen and J. Ping, Phys. Rev. D 98, 054022 (2018).

[36] C. Deng, H. Chen, and J. Ping, Eur. Phys. J. A 56, 9 (2020).

[37] W. Park, S. Noh, and S. H. Lee, Nucl. Phys. A983, 1 (2019).

[38] G. Yang, J. Ping, and J. Segovia, Phys. Rev. D 101, 014001 (2020).

[39] H. Huang and J. Ping, Eur. Phys. J. C 79, 556 (2019).

[40] E. Hernndez, J. Vijande, A. Valcarce, and J.-M. Richard, Phys. Lett. B 800, 135073 (2020).

[41] Y. Tan, W. Lu, and J. Ping, Eur. Phys. J. Plus 135, 716 (2020).

[42] Q.-F. L, D.-Y. Chen, and Y.-B. Dong, Phys. Rev. D 102, 034012 (2020).

[43] F. S. Navarra, M. Nielsen, and S. H. Lee, Phys. Lett. B 649 , 166 (2007).

[44] M.-L. Du, W. Chen, X.-L. Chen, and S.-L. Zhu, Phys. Rev. D 87, 014003 (2013).

[45] W. Chen, T. Steele, and S.-L. Zhu, Phys. Rev. D 89, 054037 (2014).

[46] Z.-G. Wang, Acta Phys. Pol. B 49, 1781 (2018).
[47] W. Chen, H.-X. Chen, X. Liu, T. Steele, and S.-L. Zhu, Phys. Rev. D 95, 114005 (2017).

[48] S. Agaev, K. Azizi, B. Barsbay, and H. Sundu, Phys. Rev. D 99, 033002 (2019).

[49] S. Agaev, K. Azizi, B. Barsbay, and H. Sundu, Phys. Rev. D 101, 094026 (2020).

[50] S. Agaev, K. Azizi, and H. Sundu, Nucl. Phys. B951, 114890 (2020).

[51] S. Agaev, K. Azizi, and H. Sundu, Phys. Rev. D 100, 094020 (2019).

[52] H. Sundu, S. Agaev, and K. Azizi, Eur. Phys. J. C 79, 753 (2019).

[53] S. Agaev, K. Azizi, B. Barsbay, and H. Sundu, arXiv: 2002.04553.

[54] Q.-N. Wang and W. Chen, Eur. Phys. J. C 80, 389 (2020).

[55] L. Tang, B.-D. Wan, K. Maltman, and C.-F. Qiao, Phys. Rev. D 101, 094032 (2020).

[56] M. A. Shifman, A. Vainshtein, and V. I. Zakharov, Nucl. Phys. B147, 385 (1979).

[57] M. A. Shifman, A. Vainshtein, and V. I. Zakharov, Nucl. Phys. B147, 448 (1979).

[58] K. Ackerstaff et al. (OPAL Collaboration), Eur. Phys. J. C 7, 571 (1999).

[59] M. Davier, A. Hcker, B. Malaescu, C.-Z. Yuan, and Z. Zhang, Eur. Phys. J. C 74, 2803 (2014).

[60] D. Boito, M. Golterman, M. Jamin, A. Mahdavi, K. Maltman, J. Osborne, and S. Peris, Phys. Rev. D 85, 093015 (2012).

[61] D. Boito, M. Golterman, K. Maltman, J. Osborne, and S. Peris, Phys. Rev. D 91, 034003 (2015).

[62] V. Abazov et al. (D0 Collaboration), Phys. Rev. Lett. 117, 022003 (2016).

[63] V. M. Abazov et al. (D0 Collaboration), Phys. Rev. D 97, 092004 (2018).

[64] R. Aaij et al. (LHCb Collaboration), Phys. Rev. Lett. 117, 152003 (2016); 118, 109904(A) (2017).

[65] A. M. Sirunyan et al. (CMS Collaboration), Phys. Rev. Lett. 120, 202005 (2018).

[66] T. Aaltonen et al. (CDF Collaboration), Phys. Rev. Lett. 120, 202006 (2018).

[67] M. Aaboud et al. (ATLAS Collaboration), Phys. Rev. Lett. 120, 202007 (2018).

[68] G. K. C. Cheung, C. E. Thomas, J. J. Dudek, and R. G. Edwards (Hadron Spectrum Collaboration), J. High Energy Phys. 11 (2017) 033.

[69] D. C. Moore and G. T. Fleming, Phys. Rev. D 74, 054504 (2006).

[70] C. Michael and I. Teasdale, Nucl. Phys. B215, 433 (1983).

[71] M. Luscher and U. Wolff, Nucl. Phys. B339, 222 (1990).

[72] B. Blossier, M. Della Morte, G. von Hippel, T. Mendes, and R. Sommer, J. High Energy Phys. 04 (2009) 094.

[73] B. Hrz and A. Hanlon, Phys. Rev. Lett. 123, 142002 (2019).

[74] A. Billoire, E. Marinari, and G. Parisi, Phys. Lett. B 162, 160 (1985).

[75] R. Gupta, G. Guralnik, G. W. Kilcup, and S. R. Sharpe, Phys. Rev. D 43, 2003 (1991).

[76] R. Hudspith (RBC, UKQCD Collaborations), Comput. Phys. Commun. 187, 115 (2015). 
[77] D. Daniel, R. Gupta, G. W. Kilcup, A. Patel, and S. R. Sharpe, Phys. Rev. D 46, 3130 (1992).

[78] S. Aoki, M. Fukugita, S. Hashimoto, Y. Iwasaki, K. Kanaya, Y. Kuramashi, H. Mino, M. Okawa, A. Ukawa, and T. Yoshie (JLQCD Collaboration), Nucl. Phys. B, Proc. Suppl. 47, 354 (1996).

[79] D. Antonio et al. (RBC, UKQCD Collaborations), Phys. Rev. D 75, 114501 (2007).

[80] P. F. Bedaque, Phys. Lett. B 593, 82 (2004).

[81] C. Sachrajda and G. Villadoro, Phys. Lett. B 609, 73 (2005).

[82] Y. Aoki et al. (RBC, UKQCD Collaborations), Phys. Rev. D 83, 074508 (2011).

[83] See Appendix B for more discussion of how to obtain physical amplitudes from these correlators.

[84] T. Bhattacharya, R. Gupta, G. Kilcup, and S. R. Sharpe, Phys. Rev. D 53, 6486 (1996).

[85] S. Aoki et al. (PACS-CS Collaboration), Phys. Rev. D 81, 074503 (2010).

[86] Y. Namekawa et al. (PACS-CS Collaboration), Phys. Rev. D 87, 094512 (2013).

[87] http://luscher.web.cern.ch/luscher/openQCD/.

[88] We implemented this heavy-quark action using AVX/ FMA2 vector intrinsics directly in openQCD. Typical charm-quark propagator inversions are comparable to those of our strange-quark propagators.

[89] M. Luscher, Comput. Phys. Commun. 156, 209 (2004).

[90] M. Luscher, J. High Energy Phys. 12 (2007) 011.

[91] M. Luscher, J. High Energy Phys. 07 (2007) 081.
[92] S. Aoki, Y. Kuramashi, and S.-I. Tominaga, Prog. Theor. Phys. 109, 383 (2003).

[93] S. Aoki, Y. Kayaba, and Y. Kuramashi, Nucl. Phys. B697, 271 (2004).

[94] Y. Namekawa et al. (PACS-CS Collaboration), Phys. Rev. D 84, 074505 (2011).

[95] D. Mohler, C. Lang, L. Leskovec, S. Prelovsek, and R. Woloshyn, Phys. Rev. Lett. 111, 222001 (2013).

[96] G. Lepage, L. Magnea, C. Nakhleh, U. Magnea, and K. Hornbostel, Phys. Rev. D 46, 4052 (1992).

[97] R. Bhaduri, L. Cohler, and Y. Nogami, Nuovo Cimento A 65, 376 (1981).

[98] S. Godfrey and N. Isgur, Phys. Rev. D 32, 189 (1985).

[99] S. Capstick and N. Isgur, AIP Conf. Proc. 132, 267 (1985).

[100] S. Aoki et al. (Flavour Lattice Averaging Group), Eur. Phys. J. C 80, 113 (2020).

[101] R. Lewis and R. Woloshyn, Phys. Rev. D 79, 014502 (2009).

[102] S. Groote and J. Shigemitsu, Phys. Rev. D 62, 014508 (2000).

[103] O. H. Nguyen, K.-I. Ishikawa, A. Ukawa, and N. Ukita, J. High Energy Phys. 04 (2011) 122.

[104] R. Dowdall et al. (HPQCD Collaboration), Phys. Rev. D 85, 054509 (2012).

[105] A. V. Manohar, Phys. Rev. D 56, 230 (1997).

[106] A. V. Manohar and M. B. Wise, Nucl. Phys. B399, 17 (1993).

[107] A. Czarnecki, B. Leng, and M. Voloshin, Phys. Lett. B 778, 233 (2018). 